Pacific

Journal of

Mathematics

QUADRATIC FORMS OVER RATIONAL FUNCTION FIELDS IN CHARACTERISTIC 2

ROBERTO ARAVIRE AND BILl JACOB 


\title{
QUADRATIC FORMS OVER RATIONAL FUNCTION FIELDS IN CHARACTERISTIC 2
}

\author{
ROBERTO ARAVIRE AND BILL JACOB
}

\begin{abstract}
A basic result of Milnor and Scharlau determines the Witt ring of rational function fields $W k(x)$ whenever char $k \neq 2$. An analogous result is obtained here for the Witt group of quadratic forms $W_{q} \mathscr{F}_{F}(x)$, where $\mathscr{F}_{F}$ is a field of characteristic 2 . This generalizes earlier work by the authors where $\mathscr{F}$ was assumed to be perfect.
\end{abstract}

Milnor's determination [1970] of the Witt ring of a rational function field in terms of the Witt rings of the finite extensions of the base field is a fundamental result in the algebraic theory of quadratic forms, and was complemented by Scharlau's reciprocity law (see [Lam 1973] or [Scharlau 1972]). Here we give an analogue of these results for the Witt group of rational function fields in characteristic 2, extending earlier work where the base field was assumed to be perfect [Aravire and Jacob 2004].

All our fields will have characteristic 2 . We use the notation $\mathscr{F}$ for the base field of our rational function field $F=\mathscr{F}(x)$. Whenever $p \in \mathscr{F}[x]$ is monic and irreducible, we denote by $\mathscr{F}(x)_{p}$ the completion at the discrete valuation $v_{p}: \mathscr{F}(x) \rightarrow \mathbb{Z}$ determined by $p$. Similarly, we denote by $\mathscr{F}(x)_{\frac{1}{x}}$ the completion at the $\frac{1}{x}$-adic (or infinite) valuation $v_{\frac{1}{x}}: \mathscr{F}(x) \rightarrow \mathbb{Z}$. We use $W_{q} F$ and $W F$ to denote the Witt group and Witt ring of $F$, and we follow the standard notation. In particular, $[a, b]$ denotes the Witt class of quadratic form $a x^{2}+x y+b y^{2}$. These classes form an additive set of generators for $W_{q} F$, and $\langle a\rangle$ denotes the 1-dimensional symmetric bilinear form $(x, y) \mapsto a x y$. The symbol [ , ] is biadditive and $W_{q} F$ is a $W F$-module via the action $\langle a\rangle[c, d]=\left[a c, a^{-1} c\right]$. This means that $W_{q} F$ is also generated by the forms $\langle a\rangle[1, b]$ and when considering such an element we will refer to $a$ as being in the multiplicative slot and $b$ as being in the additive slot. We use the standard notation $I^{n} F$ for the $n$-th power of the fundamental ideal in $W F$, so that $I^{n} W_{q} F$ is generated by the forms $\left\langle a_{1}, a_{2}, \ldots, a_{n}\right\rangle[1, b]$. Arason [1979, Satz 8] gave a generator-relation description of $W_{q} F$ as a $W F$-module, and we use these relations throughout. We frequently use what we call the fundamental relation,

MSC2000: 11E04.

Keywords: Witt group, rational function fields, characteristic two.

Both authors acknowledge support from Fondecyt. 
$\langle a+b\rangle[1, c]=\langle a\rangle[1, a c /(a+b)]+\langle b\rangle[1, b c /(a+b)]$, which shows how addition in the multiplicative slot can be distributed across a sum of forms.

A key component of the classical Milnor-Scharlau sequence is the second residue homomorphism $\partial_{p}: W F \rightarrow W \bar{F}_{p}$, where $\bar{F}_{p}$ is the residue class field of a discrete valuation $v_{p}: F \rightarrow \mathbb{Z}$. This map has proved to be of considerable importance in quadratic form theory. For example, if $X$ is a variety defined over $F$, the kernel under all $\partial_{p}: W F(X) \rightarrow W \bar{F}_{p}$ is the unramified Witt group of $X$, which when $X$ is a nonsingular curve coincides with the Witt group of $X$. This paper gives the appropriate version of $\partial_{p}$ in characteristic two, and in a subsequent paper this work is applied to the study of Witt groups of curves in characteristic two.

Whenever $v_{p}: F \rightarrow \mathbb{Z}$ is a discrete valuation, we set

$$
W_{1} F_{p}:=\operatorname{coker}\left(W_{q} \bar{F}_{p} \rightarrow W_{q} F_{p}\right),
$$

where the map is induced by a Teichmüller lifting $\bar{F}_{p} \hookrightarrow F_{p}$. We show in Corollary 1.7 that the group $W_{1} F_{p}$ is independent of the choice of a Teichmüller lifting. For such a lifting we define the second residue map $\partial_{p}: W_{q} F \rightarrow W_{1} F_{p}$ to be the composite map induced by inclusion and projection:

$$
W_{q} F \rightarrow W_{q} F_{p} \rightarrow W_{1} F_{p} .
$$

We are also able to identify a quotient of $W_{1} F_{p}$ with $W_{q} \bar{F}_{p}$ where, when $p \in \mathscr{F}[x]$ is an irreducible polynomial, we have $\bar{F}_{p} \cong \mathscr{F}[x] /(p)$. Using this we obtain a version of Scharlau's transfer $s_{p}^{*}: W_{1} F_{p} \rightarrow W_{q} \mathscr{F}$ as a composite of maps $W_{1} F_{p} \rightarrow$ $W_{q} \mathscr{F}[x] /(p) \rightarrow W_{q} \mathscr{F}$, where the latter map is the same transfer used by Scharlau. Both maps $\partial_{p}$ and $s_{p}^{*}$ are analogous to the classical maps, but as they depend upon choices of Teichmüller liftings and of subgroups of $W_{1} F_{p}$, these selections must be made to meet certain compatibility requirements for our main result to hold.

With this notation, the main result of this paper is the following.

Theorem 6.2 (Analogue of the Milnor-Scharlau Sequence). Suppose that $\mathscr{F}$ is a field of characteristic 2 and $F=\mathscr{F}(x)$ is a rational function field in one variable over F. There exists a compatible collection of second residue and transfer maps that fit into an exact sequence

$$
0 \longrightarrow W_{q} \mathscr{F} \longrightarrow W_{q} F \stackrel{\bigoplus \partial_{p}}{\longrightarrow} \bigoplus_{p, \frac{1}{x}} W_{1} F_{p} \stackrel{\bigoplus s_{p}^{*}}{\longrightarrow} W_{q} \mathscr{F} \longrightarrow 0,
$$

where the direct sum is taken over discrete valuations on $F$.

We now provide an overview of the proof. As we do this we will recall the main features of the proof in the classical case in order to illustrate the similarities and differences. When char $F \neq 2$ and $F$ is complete with respect to a discrete valuation $v: F \rightarrow \mathbb{Z}$, a well-known result of Springer shows that $W F \cong W \bar{F} \oplus$ 
$\langle\pi\rangle W \bar{F}$, where $\pi$ is a uniformizing parameter for $v$. This decomposition enables one to construct the second residue map and the transfer in the Milnor-Scharlau sequence. When char $F=2$ and $F$ is complete it is first necessary for us to compute the Witt group $W_{q} F$. This is the main objective of Section 1. The main result proved there, Theorem 1.3, shows that $W_{q} F \cong W_{q} \bar{F} \oplus \mathscr{R} \oplus\langle\pi\rangle W_{q} \bar{F}$, where again $\pi$ is a uniformizing parameter. The subgroup $\mathscr{R}$ is quite large and although its description depends upon choosing a 2-basis for $F$ and a Teichmüller lifting $\bar{F} \hookrightarrow$ $F$ it has adequate uniqueness properties. (When $\mathscr{F}$ is perfect, then there is a unique Teichmüller lifting, however in general, such lifts depend upon the choice of a 2basis for $\mathscr{F}$. See [Schilling 1950, p. 236] for details.) This decomposition shows that $W_{1} F \cong \mathscr{R} \oplus\langle\pi\rangle W_{q} \bar{F}$ and enables us to define both the second residue and Scharlau transfer maps needed for the main theorem.

After defining the second residue maps, Milnor's proof requires a filtration $L_{0} \subset$ $L_{1} \subset L_{2} \subset \cdots \subset W F$, where by $L_{d} \subset W F$ he considered the subgroup generated by all $\langle f\rangle$, where $f$ is a polynomial of degree at most $d$. He then proves a key result, namely that the successive quotients $L_{d} / L_{d-1}$ for $d \geq 1$ are isomorphic to the direct sum of groups $\bigoplus_{\operatorname{deg} p=d} W \bar{F}_{p}$. To do this he shows there is a well defined splitting of the sum of induced maps $\bigoplus_{\operatorname{deg} p=d} \partial_{p}: L_{d} / L_{d-1} \rightarrow \bigoplus_{\operatorname{deg} p=d} W \bar{F}_{p}$. In Section 2 we use the same idea and notation, except that our $L_{d}$ are generated by the forms $\langle f\rangle\left[1, h / u^{e}\right]$, where now both $f$ and $u$ have degree at most $d$ in $\mathscr{F}[x]$ and $h \in \mathscr{F}[x]$ is arbitrary. These forms are needed for two reasons. First $W_{q} F$ has as generators 2-dimensional forms, and second, the quotients $h / u^{e}$ are needed to take into account all the extra stuff in $\mathscr{R}$. In the following section, Theorem 3.5 gives the exact analogue of Milnor's key result, namely that the map

$$
\bigoplus_{\operatorname{deg} p=d} \partial_{p}: L_{d} / L_{d-1} \rightarrow \bigoplus_{\operatorname{deg} p=d} W_{1} \bar{F}_{p}
$$

is a split isomorphism.

To prove the latter result we must take several detours. First there is the complexity introduced by the existence of different ways to extend a 2-basis for $\mathscr{F}$ to a 2-basis for $F$ and $F_{p}$. If $p$ is separable, one can either add $x$ or $p$, with $x$ the natural choice for the rational function field $F$ and with $p$ the natural choice for $F_{p}$. When $p$ is not separable, we have to specify which element we choose to omit from the 2-basis of $\mathscr{F}$ and then we must add both $x$ and $p$ to form a 2-basis for $F_{p}$. Since the $\partial_{p}$ relate $W_{q} F$ to $W_{1} F_{p}$, we need to be able to relate these choices. The bulk of Section 2 accomplishes this, by establishing the equivalence of different generating sets for the $L_{d}$ in Lemma 2.5 and Proposition 2.8.

A second detour provides a generator-relation description of $W_{q} F$ (Theorem 3.3) needed to prove that the splitting maps are well defined (Lemma and Definition 3.4). The proof of the splitting is similar to that of the classical case, but is 
complicated again by the fact that 2-bases and Teichmüller liftings have to be selected carefully and in a compatible fashion. The details of these choices are set up in the discussion that follows Lemma 3.1. Finally one has to deal with the structure of $L_{0}$, which is just $W \mathscr{F}$ in the classical case. In our case it is generated by forms with polynomials $f, g \in \mathscr{F}[x]$ in the additive slots of binary forms, $[f, g]$. The result in Theorem 3.6 is that $L_{0}$ is described by an exact sequence $0 \rightarrow W_{q} \mathscr{F} \rightarrow L_{0} \rightarrow W_{1} F_{\frac{1}{x}} \rightarrow W_{q} \mathscr{F} \rightarrow 0$.

When Theorems 3.5 and 3.6 are combined with the definitions, we obtain a version of what Milnor did, namely that the sequence in Theorem 6.2 is exact if truncated to

$$
0 \longrightarrow W_{q} \mathscr{F} \longrightarrow W_{q} F \stackrel{\bigoplus \partial_{p}}{\longrightarrow} \bigoplus_{p, \text { finite }} W_{1} F_{p} \oplus\left(W_{1} F_{\overline{1}} /\langle x\rangle W \mathscr{F}\right) \longrightarrow 0,
$$

where the reciprocity law provided by the transfer is omitted. However, because the reciprocity law has important applications, we continue with its development in subsequent sections. Section 4 is devoted to defining the transfer maps. The subgroups of $W_{1} F_{p}$ needed to define the maps are given in Definition 4.1 and are selected in a compatible way to ensure that the resulting $s_{p}^{*}$ vanish on the subgroup $\mathscr{R} \subset W_{1} F_{p}$. The definition of $s_{p}^{*}$ when $p$ fails to be separable, Definition 4.3(ii), is adjusted to take into account the change in the 2-basis resulting from the failure of the 2-basis of $\mathscr{F}$ to extend to $F_{p}$. In this case the exact terms necessary to make the reciprocity law work are added to the transfer of the residue form.

Having defined the $s_{p}^{*}$, we check the reciprocity law for elements of $L_{0}+\langle p\rangle L_{0}$ (Theorem 5.4). This requires computing the ordinary transfer $t_{p}^{*}: W_{q} \mathscr{F}[x] /(p) \rightarrow$ $W_{q} \mathscr{F}$ on generators $\left[\lambda_{1} x^{i}, \lambda_{2} x^{j}\right]$ of $W_{q} \mathscr{F}[x] /(p)$. There are quite a few cases to consider, but it is a straightforward computation. With this result, the main theorem, with the reciprocity law in general, is proved in Section 6, where the final stages of the proof consist of checking that the definitions involved in setting up $W_{1} F_{p}$ and the $s_{p}^{*}$ are arranged properly to ensure cancellation of the appropriate terms. Although the definition of $s_{p}^{*}$ is based on the same linear functional as in the classical case, this portion of the paper differs from the approach in that case. Because of the additive nature of generators for $W_{q} F$ we are able to reduce to forms that vanish on all but two $\partial_{p}$ 's, and therefore we don't have to consider more complex transfers from algebras such as $\mathscr{F}[x] /\left(p_{1} p_{2} \cdots p_{n}\right)$, as did Scharlau.

\section{Local information}

If $F$ has characteristic 2, a collection of elements $t_{1}, t_{2}, \ldots, t_{n} \in F$ is said to form a 2-basis of $F$ if we have a strictly increasing sequence of subfields

$$
F^{2} \varsubsetneqq F^{2}\left(t_{1}\right) \varsubsetneqq F^{2}\left(t_{1}, t_{2}\right) \varsubsetneqq \cdots \varsubsetneqq F^{2}\left(t_{1}, t_{2}, \ldots, t_{n}\right)=F .
$$


A field $F$ can have many different 2-bases; if $\left[F: F^{2}\right]=2^{n}$, every 2-basis has exactly $n$ elements. We will assume that fields in this paper have finite 2-bases, since our main results are readily reduced to this case.

For fixed $n$ we denote by $T$ the set of $n$-tuples $I=\left(i_{1}, i_{2}, \ldots, i_{n}\right)$, where $i_{j} \in\{0,1\}$ for all $j$. We order $T$ lexicographically, with minimal element $O:=$ $(0,0, \ldots, 0)$, then $(1,0, \ldots, 0)$, then $(0,1,0, \ldots, 0)$, and so forth. It will be convenient to add elements of $T$ as in the $\mathbb{Z} / 2 \mathbb{Z}$-vector space $(\mathbb{Z} / 2 \mathbb{Z})^{n}$ and let $T_{0}$ denote the nonzero elements of $T$. Whenever $t_{1}, t_{2}, \ldots, t_{n} \in F$ and $I \in T$, we abbreviate $t_{1}^{i_{1}} t_{2}^{i_{2}} \cdots t_{n}^{i_{n}}$ by $t^{I}$. In this notation, whenever $t_{1}, t_{2}, \ldots, t_{n}$ form a 2-basis for $F$ and $f \in F$, there exist unique elements $x_{I} \in F$ indexed by $I \in T$ such that

$$
f=\sum_{I \in T} t^{I} x_{I}^{2}
$$

For the remainder of this section we assume that $v: F \rightarrow \mathbb{Z}$ is a complete discrete-valued field of characteristic 2 with residue class field $\bar{F}$ and uniformizing parameter $\pi$. We assume that $t_{1}, t_{2}, \ldots, t_{n-1}$ are units in $F$ whose residues form a 2-basis for $\bar{F}$. Since $v$ is complete and discrete, we know that $t_{1}, t_{2}, \ldots, t_{n-1}, \pi$ form a 2-basis for $F$. We will use the notation $t^{I}$, where $I=\left(I_{1}, I_{2}, \ldots, I_{n}\right) \in T$, to represent elements in this 2-basis:

$$
t^{I}=t_{1}^{I_{1}} t_{2}^{I_{2}} \cdots t_{n-1}^{I_{n-1}} \pi^{I_{n}} .
$$

From [Schilling 1950, pp. 230-238] we also know that there is a unique monomorphism of fields $\rho: \bar{F} \hookrightarrow F$ with $\rho\left(\bar{t}_{i}\right)=t_{i}$ for $i=1,2, \ldots, n-1$. Since we will regard this map as an inclusion, we will drop the residue bars from the $t_{i}$ and view $t_{1}, t_{2}, \ldots, t_{n-1}$ as lying in $\bar{F} \subset F$. With these conventions, $v\left(t^{J}\right)=J_{n} \in\{0,1\}$ for all $J \in T$.

Since $F$ is complete, we can view $F=\bar{F}((\pi))$ as formal Laurent series in its fixed uniformizing parameter $\pi$. We let $R:=\bar{F}\left[\pi^{-1}\right] \subset F$ be the "backwards" polynomial ring, and with this, if $f \in F$ then there exists a unique $r \in \pi^{-1} R$ with $v(f-r) \geq 0$. Moreover, every element $r \in \pi^{-1} R$ can be uniquely expressed as $r=\sum_{I \in T} t^{I} r_{I}^{2}$, where $r_{I} \in R$.

Definition 1.1. We set $\mathscr{R}$ to be the subgroup of $W_{q} F$ of all forms

$$
\sum_{I \in T}\left\langle t^{I}\right\rangle\left[1, \sum_{J, J+I>I} t^{J} r_{I, J}^{2}\right] \in \mathscr{R} \quad \text { with } r_{I, J} \in \pi^{-1} R \text {. }
$$

Whenever $v(a)>0$ (that is, $a \in \bar{F}((\pi)))$, we must have $a \in \wp(F)$ since $F$ is complete with respect to $v$; consequently, $[1, a]=0 \in W_{q} F$. We will use this fact repeatedly. When $r \in \pi^{-1} R$, however, we are in the opposite situation, since then $v(r)<0$; in this case, if $v(r)$ is odd or the lead coefficient is not a square in $\mathscr{F}$, then $r$ cannot lie in $\wp(F)$. This is why the module $\mathscr{R}$ is of interest. 
Lemma 1.2. Every element $\phi \in W_{q} F$ can be expressed as $\phi=\sum_{I \in T}\left\langle t^{I}\right\rangle\left[1, A_{I}\right]$, where $A_{I}=\sum_{J, J+I>I} t^{J} r_{I, J}^{2}$ and $r_{I, J} \in F$.

Proof. Applying the $W_{q}$-relations we know that every element of $W_{q} F$ is a sum of elements $\left\langle t^{I}\right\rangle\left[1, t^{J} b^{2}\right]$ for $b \in F$. Applying the $W_{q}$-relation $\langle t\rangle[1, a]=\langle t a\rangle[1, a]$ we find for $I, J$ with $I+J<I$ that $\left\langle t^{I}\right\rangle\left[1, t^{J} b^{2}\right]=\left\langle t^{I+J}\right\rangle\left[1, t^{J} b^{2}\right]$, and when $I=J$ we find that $\left\langle t^{I}\right\rangle\left[1, t^{I} b^{2}\right]=\langle 1\rangle\left[1, t^{I} b^{2}\right]$. Hence every element of $\phi \in W_{q} F$ can be expressed as $\phi=\sum_{I \in T}\left\langle t^{I}\right\rangle\left[1, \sum_{J, J+I>I} t^{J} b_{I, J}^{2}\right]$, with $b_{I, J} \in F$. (In fact this much is true for any 2-basis of any field $F$ of characteristic 2.) The statement for $\mathscr{R}$ follows applying this same argument to that case.

Theorem 1.3. Suppose that $v: F \rightarrow \mathbb{Z}$ is a complete discrete valued field of characteristic 2 with residue field $\bar{F} \subset F$ and uniformizing parameter $\pi$. Then every class $\phi \in W_{q} F$ can be expressed uniquely as

$$
\phi=\phi_{1} \perp \psi \perp\langle\pi\rangle \phi_{2},
$$

where $\phi_{1}, \phi_{2} \in W_{q} \bar{F}$ and $\psi=\sum_{I \in T}\left\langle t^{I}\right\rangle\left[1, \sum_{J, J+I>I} t^{J} r_{I, J}^{2}\right] \in \mathscr{R}$ with $r_{I, J} \in$ $\pi^{-1} R$. The classes of $\phi_{1}, \phi_{2}$ and the $r_{I, J}$ are uniquely determined by $\phi$. In particular, there is a split exact sequence

$$
0 \rightarrow W_{q} \bar{F} \rightarrow W_{q} F \rightarrow\left(\mathscr{R} \oplus\langle\pi\rangle W_{q} \bar{F}\right) \rightarrow 0 .
$$

Proof. Consider $\sum_{I \in T}\left\langle t^{I}\right\rangle\left[1, \sum_{J, J+I>I} t^{J} b_{I, J}^{2}\right] \in W_{q} F$. Since $F$ is complete and discretely valued, we can express each $b_{I, J}$ as $r_{I, J}+\bar{f}_{I, J}+b_{I, J}^{\prime}$, where $r_{I, J} \in \pi^{-1} R$, $\bar{f}_{I, J} \in \bar{F} \subset F$ and $v\left(b_{I, J}^{\prime}\right)>0$. Since $v\left(t^{J}\right) \geq 0$ we have $t^{J} b_{I, J}^{\prime}{ }^{2} \in \wp(F)$ and hence $\left[1, t^{J} b_{I, J}^{\prime}{ }^{2}\right]=0$. We observe:

- If $J_{n}=0$ then $t^{J} \in \bar{F}$ and we have $t^{J} r_{I, J}^{2} \in \pi^{-1} R$ while $t^{J} \bar{f}_{I, J}^{2} \in \bar{F} \subset F$. When $I_{n}=0$ we have $\left\langle t^{I}\right\rangle\left[1, t^{J} \bar{f}_{I, J}^{2}\right] \in W_{q} \bar{F}$, and when $I_{n}=1$ we have $\left\langle t^{I}\right\rangle\left[1, t^{J} \bar{f}_{I, J}^{2}\right] \in$ $\langle\pi\rangle W_{q} \bar{F}$. So

$$
\left\langle t^{I}\right\rangle\left[1, t^{J} b_{I, J}^{2}\right]=\left\langle t^{I}\right\rangle\left[1, t^{J} r_{I, J}^{2}\right]+\left\langle t^{I}\right\rangle\left[1, t^{J} \bar{f}_{I, J}^{2}\right] \in W_{q} \bar{F}+\mathscr{R}+\langle\pi\rangle W_{q} \bar{F}
$$

in this case.

- If $J_{n}=1$, then we know $v\left(t^{J}\right)=1$ and consequently $t^{J} r_{I, J}^{2} \in \pi^{-1} R$ while $v\left(t^{J} \bar{f}_{I, J}^{2}\right)>0$, so $\left[1, t^{J} \bar{f}_{I, J}^{2}\right]=0$. Therefore, $\left\langle t^{I}\right\rangle\left[1, t^{J} b_{I, J}^{2}\right]=\left\langle t^{I}\right\rangle\left[1, t^{J} r_{I, J}^{2}\right] \in \mathscr{R}$ in this case.

Altogether this shows that every element of $\phi \in W_{q} F$ can be expressed as $\phi=$ $\phi_{1}+\psi+\langle\pi\rangle \phi_{2}$, with $\phi_{1}, \phi_{2} \in W_{q} \bar{F}$ and $\psi \in \mathscr{R}$.

To prove the uniqueness assertions and the exactness of the sequence we need a bit more notation. We denote by $n^{s}$ the set of all subsets of $\{1,2, \ldots, n\}$ containing $s$ elements. For any $I=\left(I_{1}, I_{2}, \ldots, I_{n}\right) \in T$ we use $\tilde{I}$ to denote $\left\{j \mid I_{j} \neq 0\right\}$. Note 
that $\tilde{I} \in n^{s}$ for some $s=0,1, \ldots, n$, and that any subset $\tilde{I} \in n^{s}$ is determined by a unique $I \in T$. For any subset $S \subseteq\{1,2, \ldots, n\}$ we write

$$
\left\langle t_{S}\right\rangle=\bigotimes_{i \in S}\left\langle t_{i}\right\rangle
$$

where, when $S=\varnothing,\left\langle t_{\varnothing}\right\rangle=\langle 1\rangle$ by convention. Whenever $I \in T$, we define $\ell(I)=\max (\tilde{I})$. Finally, we define $[\tilde{I}]_{0}:=\{J \in T \mid J \neq(0,0, \ldots, 0)$ and $\tilde{J} \subseteq \tilde{I}\}$ and $\left[\tilde{I}^{c}\right]:=\{J \in T \mid \tilde{J} \cap \tilde{I}=\varnothing\}$.

Lemma 1.4 [Aravire and Jacob 1996, Lemma 1.6]. Suppose that $t_{1}, t_{2}, \ldots, t_{n}$ are 2-independent in a field $F$ and let $a_{\tilde{I}} \in F$. Suppose

$$
q=\sum_{\tilde{I} \in n^{s}} \ll t_{\tilde{I}} 》\left[1, a_{\tilde{I}}\right] \in I^{s+1} W_{q} F .
$$

Then each

$$
a_{\tilde{I}} \in\left(\wp(F)+\sum_{J \in[\tilde{I}]_{0}+\left[\tilde{I}^{c}\right]} t^{J} F^{2}\right)=\left(\wp(F)+\sum_{J, \tilde{J} \cap \tilde{I} \neq \varnothing} t^{J} F^{2}\right)
$$

The next result is a modification of [Aravire and Jacob 1996, Proposition 1.7].

Proposition 1.5. Suppose that $v: F \rightarrow \mathbb{Z}$ is a complete discrete valued field and $t_{1}, t_{2}, \ldots, t_{n}$ are as above. Suppose

$$
q=\sum_{\tilde{I} \in n^{s}} 《 t_{\tilde{I}} 》\left[1, a_{\tilde{I}}\right] \in I^{s+1} W_{q} F,
$$

where $a_{\tilde{I}} \in \sum_{J+I>I} t^{J}\left(\pi^{-1} R\right)^{2}$. Then $a_{\tilde{I}}=0$ for each $I$.

Proof. Assume the contrary. Let $M$ be the maximal index among the $I$ with $\tilde{I} \in n^{s}$ and $a_{\tilde{I}} \neq 0$. We express $a_{\tilde{M}}$ as a sum $\sum_{K+M>M} t^{K} A_{K, M}^{2}$, where each $A_{K, M} \in \pi^{-1} R$. Since $a_{\widetilde{M}} \neq 0$, there is some $J$ with $J+M>M$ and $t^{J} A_{J, M}^{2} \neq 0$. The result will be proved when we derive the contradiction that $t^{J} A_{J, M}^{2}=0$.

Since $J+M>M$ we have $\ell(J) \notin \widetilde{M}$. We denote by $t_{1}^{\prime}, t_{2}^{\prime}, \ldots, t_{n}^{\prime}$ the 2-basis obtained from $t_{1}, t_{2}, \ldots, t_{n}$ by replacing $t_{\ell(J)}$ by $t^{J}$. Then, since $\ell(J) \notin \tilde{M}$, we have $\left\langle t_{\tilde{M}}\right\rangle=\left\langle t_{\tilde{M}}^{\prime} 》\right.$. Also, we have

$$
\left\langle t_{\ell(J)}^{\prime}\right\rangle=\left\langle t^{J} 》 \equiv \sum_{j \in \tilde{J}}\left\langle t^{j}\right\rangle\left(\bmod I^{2} F\right)\right.
$$


We now suppose that $K \in T, \tilde{K} \in n^{s}$, and $\ell(J) \in \tilde{K}$. We express $\tilde{K}$ as $\{\ell(J)\} \cup \tilde{Q}$ for $\tilde{Q} \in n^{s-1}$. Computing in $W F$ modulo $I^{s+1} F$ we have

$$
\begin{aligned}
\left\langle t_{\tilde{K}}^{\prime} 》\right. & =\left\langle t_{\tilde{Q}}^{\prime} 》\left\langle t_{\ell(J)}^{\prime}\right\rangle=\left\langle t_{\tilde{Q}} 》\left\langle t^{J}\right\rangle \equiv \sum_{j \in \tilde{J}}\left\langle t_{\tilde{Q}}\right\rangle\left\langle t_{j}\right\rangle\right.\right. \\
& \equiv\left\langle t_{\tilde{K}}\right\rangle+\sum_{\substack{j \in \tilde{J} \\
j \neq \ell(J)}}\left\langle t_{\tilde{Q}}\right\rangle\left\langle t_{j}\right\rangle \equiv\left\langle t_{\tilde{K}}\right\rangle+\sum_{\substack{j \in \tilde{J}, j \neq \ell(J) \\
j \notin \tilde{Q}}}\left\langle t_{\tilde{Q}}\right\rangle\left\langle t_{j}\right\rangle .
\end{aligned}
$$

The conditions $j \in \tilde{J}, j \neq \ell(J)$, and $j \notin \tilde{Q}$, are equivalent to the single condition $j \in \tilde{J}-\tilde{K}$. So, as each such $j<\ell(J)$ we find $\left\langle t_{\tilde{Q}} 》\left\langle t_{j}\right\rangle=\left\langle t_{\tilde{Q}}^{\prime} 》\left\langle t_{j}^{\prime}\right\rangle=\left\langle t_{\tilde{L}}^{\prime}\right\rangle\right.\right.$ for some $L \in T$ with $L<K$. Altogether this shows that whenever $K \in T, \tilde{K} \in n^{s}$, and $\ell(J) \in \tilde{K}$,

$$
\left\langlet _ { \tilde { K } } 》 \in \left\langle t_{\tilde{K}}^{\prime} 》+\left(\sum_{\substack{L \in T \\ \tilde{L} \in n^{s}, L<K}}\left\langle t_{\tilde{L}}^{\prime} 》 W F+I^{s+1} F\right) .\right.\right.\right.
$$

Expanding using (1) we can rewrite $q$ in terms of the new 2-basis involving the $t^{\prime}$. We find

$$
\begin{aligned}
q & =\sum_{\tilde{I} \in n^{s}}\left\langle t_{\tilde{I}}\right\rangle\left[1, a_{\tilde{I}}\right]=\left(\sum_{\substack{K<M \\
\tilde{K} \in n^{s}}}\left\langle t_{\tilde{K}}\right\rangle\left[1, a_{\tilde{K}}\right]\right)+\left\langle t_{\tilde{M}} 》\left[1, a_{\tilde{M}}\right]\right. \\
& \equiv\left(\sum_{\substack{K<M \\
\tilde{K} \in n^{s}}}\left\langle t_{\tilde{K}}^{\prime} 》\left[1, a_{\tilde{K}}^{\prime}\right]\right)+\left\langle t_{\tilde{M}}^{\prime} 》\left[1, a_{\tilde{M}}\right]\left(\bmod I^{s+1} W_{q} F\right),\right.\right.
\end{aligned}
$$

where the $a_{\tilde{K}}^{\prime}$ for $K<M$ are the elements of $F$ that arise in the expansion using (1) repeatedly. Observe that $a_{\widetilde{M}}$ remains unchanged when passing to the 2-basis using the $t^{\prime}$. We now apply Lemma 1.2, where the 2-basis used is the one with the $t^{\prime}$. We find that

$$
a_{\tilde{M}}=\sum_{K+M>M} t^{K} A_{K, M}^{2} \in \wp(F)+\sum_{L, \tilde{L} \cap \tilde{M} \neq \varnothing} t^{\prime L} F^{2} .
$$

When constructing the 2-basis involving $t^{\prime}$ we replaced $t_{\ell(J)}$ by $t^{J}$, which means that $t^{J}=t^{\prime J^{\prime}}$, where $\tilde{J}^{\prime}=\{\ell(J)\} \in n^{1}$. Since $\ell(J) \notin \tilde{M}$, this gives $\tilde{J}^{\prime} \cap \tilde{M}=\varnothing$. Therefore, moving all the other terms on the left side of (2) to the right we find

$$
t^{J} A_{J, M}^{2} \in \wp(F)+\sum_{I \in T_{0}, I \neq J} t^{I} F^{2} .
$$

We claim that this gives $A_{J, M}=0$. As $t^{J} A_{J, M}^{2} \in \pi^{-1} R$, if $t^{J} A_{J, M}^{2} \neq 0$ we must have $v\left(t^{J} A_{J, M}^{2}\right)=s<0$, where $s$ is even if $J_{n}=0$ and is odd if $J_{n}=1$. For $K \in T$ 
let $K^{\prime}$ be such that $K_{n}^{\prime}=0$ and $K_{i}^{\prime}=K_{i}$ for $1 \leq i<n$. With this notation,

$$
\overline{\pi^{-s} t^{J} A_{J, M}^{2}} \in t^{J^{\prime}} \bar{F}^{2} \text {. }
$$

Next, if $w=\wp(b)+\sum_{I \in T_{0}, I \neq J} t^{I} b_{I, J}^{2} \in \wp(F)+\sum_{I \in T_{0}, I \neq J} t^{I} F^{2}$ is such that $v(b)=s<0$, then

$$
\overline{p i^{-s} w} \in\left\{\begin{array}{cl}
\bar{F}^{2}+\sum_{\substack{I \in T_{0}, I \neq J \\
I_{n}=0}} t^{I} \bar{F}^{2} \text { when } s \text { is even, } \\
\sum_{\substack{I^{\prime} \in T_{0}, I \neq J \\
I_{n}=1}} t^{I^{\prime}} \bar{F}^{2} \text { when } s \text { is odd. }
\end{array}\right.
$$

In either case, because $t_{1}, t_{2}, \ldots, t_{n-1}$ is a 2 -basis for $\bar{F}$, we cannot have

$$
\overline{\pi^{-s} t^{J} A_{J, M}^{2}}=\overline{\pi^{-s} w},
$$

contrary to the assumption that $A_{J, M} \neq 0$. This proves the proposition.

We may now complete the proof of Theorem 1.3. The main task is showing that if $\psi=\sum_{I \in T}\left\langle t^{I}\right\rangle\left[1, \sum_{J, J+I>I} t^{J} r_{I, J}^{2}\right]=0 \in \mathscr{R}$ with $r_{I, J} \in \pi^{-1} R$, then each $r_{I, J}=0$. Assuming this temporarily for all such complete discrete valued fields, to prove the uniqueness statements we consider an expression $\phi=\phi_{1} \perp \psi \perp\langle\pi\rangle \phi_{2}=0$. Let $L$ be a separable finite unramified extension of $F$ chosen so that $\left(\phi_{1}\right)_{L}=0$ and $\left(\phi_{2}\right)_{L}=0$. Then $L$ is still complete and discretely valued, the 2-basis is unchanged, $R \subset R_{L}$, and we have that $\phi_{L}=\psi_{L}$. So our temporary assumption applies to $\psi_{L}=0 \in \mathscr{R}_{L}$, the $r_{I, J}$ vanish in this case, and we now have $\phi_{1} \perp\langle\pi\rangle \phi_{2}=0$. Now, by valuation theory, if both $\phi_{1}$ and $\phi_{2}$ are anisotropic over $\bar{F}$, then $\phi_{1} \perp\langle\pi\rangle \phi_{2}$ anisotropic as well, since $\pi$ is a uniformizing parameter. So this gives $\phi_{1}=\phi_{2}=0$ and the uniqueness assertion follows.

Thus we are reduced to studying $\psi=\sum_{I \in T}\left\langle t^{I}\right\rangle\left[1, \sum_{J, J+I>I} t^{J} r_{I, J}^{2}\right]=0 \in \mathscr{R}$, where we want to show that each $r_{I, J}=0$.

Lemma 1.6 [Aravire and Jacob 1996, Lemma 1.5]. Suppose that $t_{1}, t_{2}, \ldots, t_{n}$ are 2-independent in a field $F$ and $f \in F$. Then $\left\langle t_{1}, t_{2}, \ldots, t_{n} 》[1, f]=0 \in W_{q} F\right.$ if and only if

$$
f \in \wp(F)+\sum_{J \in T_{0}} t^{J} F^{2}
$$

Applying the identity in $W F$ (symmetric bilinear forms)

$$
\langle x y\rangle=\langle x x\rangle+\langle y\rangle+\langle\langle x, y\rangle,
$$

we obtain

$$
\left\langle t^{I}\right\rangle=\sum_{K, \tilde{K} \subseteq \tilde{I}}\left\langle t_{\tilde{K}}\right\rangle
$$


(recall that $\left\langle t_{\varnothing}\right\rangle=\langle 1\rangle$.) Abbreviating $a_{I}:=\sum_{J, J+I>I} t^{J} r_{I, J}^{2}$ and rewriting $\psi$ using this identity we obtain

$$
\begin{aligned}
0=\psi= & \left.\sum_{I \in T}\left\langle t^{I}\right\rangle\left[1, a_{I}\right]=\sum_{I \in T}\left(\sum_{K, \tilde{K} \subseteq \tilde{I}} \ll t_{\tilde{K}}\right\rangle\right)\left[1, a_{I}\right] \\
& =\sum_{K \in T} \ll t_{\tilde{K}} 》\left[1, \sum_{I, \tilde{K} \subseteq \tilde{I}} a_{I}\right]=\sum_{s=0, \ldots, n}\left(\sum_{\tilde{K} \in n^{s}}\left\langle t_{\tilde{K}} 》\left[1, \sum_{I, \tilde{K} \subseteq \tilde{I}} a_{I}\right]\right) .\right.
\end{aligned}
$$

By induction on $s$ we shall show that

$$
\sum_{I, \tilde{K} \subseteq \tilde{I}} a_{I}=0
$$

whenever $K \in n^{s}$. When $s=0, \tilde{K}=\varnothing$ and since $\sum_{I \in T} a_{I}$ is the Arf invariant of $q$ we find $\sum_{I \in T} a_{I} \in \wp(F)$. Since nonzero elements of $\pi^{-1} R$ have negative value, by valuation theory we find $\sum_{I \in T_{0}} t^{I}\left(\pi^{-1} R\right)^{2} \cap \wp(F)=\{0\}$ and we are done if $s=0$. Assuming the result for $1,2, \ldots, s-1$, we have

$$
\sum_{\tilde{K} \in n^{s}}\left\langle t_{\tilde{K}} 》\left[1, \sum_{I, \tilde{K} \subseteq \tilde{I}} a_{I}\right] \in I^{s+1} W_{q} F .\right.
$$

We observe that if $\tilde{K} \subseteq \tilde{I}$ and $J+I>I$, then $J+K>K$. Therefore Proposition 1.5 applies and we conclude for fixed $\tilde{K} \in n^{s}$ that

$$
\sum_{I, \tilde{K} \subseteq \tilde{I}} a_{I}=0 .
$$

Using $a_{I}=\sum_{I+J>I} t^{J} r_{I, J}^{2}$ we obtain

$$
0=\sum_{I, \tilde{K} \subseteq \tilde{I}} a_{I}=\sum_{I, \tilde{K} \subseteq \tilde{I}}\left(\sum_{I+J>I} t^{J} r_{I, J}^{2}\right)=\sum_{J \in T_{0}} t^{J}\left(\sum_{\substack{I, \tilde{K} \subseteq \tilde{I} \\ I+J>I}} r_{I, J}^{2}\right) .
$$

Since $t_{1}, t_{2}, \ldots, t_{n}$ form a 2-basis of $F$, for fixed $K, J$ we find

$$
\sum_{\substack{I, \tilde{K} \subseteq \tilde{I} \\ I+J>I}} r_{I, J}^{2}=0 .
$$

We next show that $r_{I, J}^{2}=0$ for all $I, J$ such that $I+J>I$. We proceed by reverse induction on $\operatorname{card}(\tilde{I})$. If $\operatorname{card}(\tilde{I})=n$ we have $I=(1, \ldots, 1)$ and $I+J>J$ is impossible, so the conclusion is vacuous. Now suppose the desired conclusion is known for all $I$ with $\operatorname{card}(\tilde{I})>r$. Fix some $K$ with $\operatorname{card}(\tilde{K})=r$ and some $J$ with $J+K>K$. If $I \neq K$, and if $\tilde{K} \subset \tilde{I}$, we have $\operatorname{card}(\tilde{I})>r$. Our inductive hypothesis 
implies that $r_{I, J}^{2}=0$ for these $I, J$, and since these are all but one summand of (3), we find that $r_{K, J}^{2}=0$ as well. This completes the induction. The definitions give $a_{I}=0$ and the proof of Theorem 1.3 is complete.

Since the ring $R=\bar{F}\left[\pi^{-1}\right] \subset F$ is simply a polynomial ring over the residue field $\bar{F}$ of $F$, the choice of the lift to $F$ of the 2-basis $t_{1}, t_{2}, \ldots, t_{n-1}$ of $\bar{F}$ does not affect the isomorphism type of $R$ or $R$. This, together with the uniqueness results, implies:

Corollary 1.7. Suppose that $v: F \rightarrow \mathbb{Z}$ is a complete discrete valued field of characteristic 2 with residue field $\bar{F}$ and uniformizing parameter $\pi$. Then up to isomorphism, the submodule $\mathscr{R}$ is independent of the choice of lift of the 2-basis $t_{1}, t_{2}, \ldots, t_{n-1}$ of $\mathscr{F}_{\text {. }}$ In particular, the cokernel

$$
W_{1} F:=\operatorname{coker}\left(W_{q} \bar{F} \rightarrow W_{q} F\right)
$$

is independent of the choice of lift of this 2-basis.

Remark 1.8. Both residue forms $\phi_{1}$ and $\phi_{2}$ in Theorem 1.3 depend upon the choice of the uniformizing parameter $\pi$.

When $F$ is complete and discretely valued, the group $W_{1} F$ defined in Corollary 1.7 will play the role of the "second residue forms" in characteristic 2 . The projection map $\partial_{v}: W_{q} F \rightarrow W_{1} F$, is the analogue of the second residue map. It is an immediate consequence of this definition that

$$
0 \longrightarrow W_{q} \bar{F} \longrightarrow W_{q} F \stackrel{\partial_{v}}{\longrightarrow} W_{1} F \longrightarrow 0
$$

is split exact. (This definition also coincides with the second residue map away from characteristic 2, for in that case Springer's Theorem gives a group isomorphism $W F \cong W \bar{F} \oplus\langle p\rangle W \bar{F}$, so $W_{1} F \cong W \bar{F}$.)

Remark 1.9. Arason [2003] has proved a result that captures all the information in Theorem 1.3. His proof uses the generator-relation structure of the Witt group. His description of $\mathscr{R}$ is different (it uses a filtration based on negative exponents of the uniformizing parameter) and his proof does not require powers of the fundamental ideal since he directly uses the generator relation structure for the Witt group.

\section{The filtration of $W_{q} \mathscr{F}(x)$}

We now denote by $\mathscr{F}_{F}$ a fixed field of characteristic 2 with 2 -basis $t_{1}, t_{2}, \ldots, t_{n}$. We study the Witt group of the field of rational functions $F=\mathscr{F}(x)$. The results of the previous section will be applied to the completions of $F$ at its discrete valuations, which are trivial on $\mathscr{F}$. Following Milnor's original approach away from characteristic 2, we also filter the Witt group $W_{q} F$ by degree. We denote by $\mathscr{F}[x]_{\leq d}$ the 
set of polynomials in $\mathscr{F}[x]$ of degree at most $d$, and by $\mathscr{F}[x]_{<d}$ those of degree less than $d$.

Definition 2.1. For $d \geq 1$, let $L_{d}$ be the subgroup of $W_{q} \mathscr{F}(x)$ generated by all forms $\langle f\rangle\left[1, h / u^{e}\right]$, where $f, u \in \mathscr{F}[x]_{\leq d}$ and $h \in \mathscr{F}[x]$. When $d=0$, let $L_{0}$ be the subgroup of $W_{q} \mathscr{F}(x)$ generated by the forms $\left[\lambda_{1} x^{i}, \lambda_{2} x^{j}\right]$, where $\lambda_{1}, \lambda_{2} \in \mathscr{F}$ and $i, j \in \mathbb{N}$.

Lemma 2.2. (i) For any polynomials $p, g, h \in \mathscr{F}[x]$ we have $\langle p\rangle[g, p h] \in L_{0}$.

(ii) For $d \geq 1, L_{d}$ is generated by the forms $\left\langle a x^{\epsilon}\right\rangle\left[1, h / u^{e}\right]$, where $a \in \mathscr{F}_{F}, \epsilon \in\{0,1\}$, $h \in \mathscr{F}[x]$ and $u$ factors as a product of elements in $\mathscr{F}[x]_{\leq d}$.

(iii) If $f, u \in \mathscr{F}[x]_{<d}$ and $p \in \mathscr{F}[x]$ then $\langle p f\rangle\left[1, p g / u^{e}\right] \in L_{d-1}$.

Proof. (i) The first statement follows from the identity $\langle a\rangle[b, c]=[a b, c / a]$. For then $\langle p\rangle[g, p h]=[p g, h]$, and using the biadditivity of the symbol $[$,$] this can$ be expressed as a sum of generators for $L_{0}$.

(ii) For the second statement, since the $u$ 's used as generators in this version are products of elements in $\mathscr{F}[x]_{\leq d}$, we can use apply partial fractions to $h / u^{e}$ together with the additivity of $[$,$] to express \left\langle a x^{\epsilon}\right\rangle\left[1, h / u^{e}\right]$ as a sum of generators of the type in specified in Definition 2.1. Conversely, given $\langle f\rangle\left[1, h / u^{e}\right]$ as in Definition 2.1, where $f, u \in \mathscr{F}[x]_{\leq d}$, and given $h \in \mathscr{F}[x]$, we write $f=\sum_{i=0}^{d-1} a_{i} x^{i}$ with $a_{i} \in \mathscr{F}$ and use the fundamental relation to express $\langle f\rangle\left[1, h / u^{e}\right]$ in the form $\sum_{i=0}^{d-1}\left\langle a_{i} x^{i}\right\rangle\left[1, h a_{i} x^{i} / f u^{e}\right]$. Since $\left\langle x^{i}\right\rangle=\left\langle x^{\epsilon_{i}}\right\rangle$, where $\epsilon_{i} \in\{0,1\}$ and $i \equiv \epsilon_{i}(\bmod$ 2 ), and since $h a_{i} x^{i} / f u^{e}=h a_{i} x^{i} f^{e-1} /(f u)^{e}$, we have a generator of the desired type.

(iii) We apply the fundamental relation, expressing $p f$ as $\sum_{i=1}^{n-1} a_{i} x^{i}$ with $a_{i} \in \mathscr{F}_{F}$, so $\langle p f\rangle\left[1, p g / u^{e}\right]=\sum_{i=1}^{n-1}\left\langle a_{i} x^{i}\right\rangle\left[1, p_{i} x^{i} p g / p f u^{e}\right]=\sum_{i=1}^{n-1}\left\langle a_{i} x^{i}\right\rangle\left[1, p_{i} x^{i} g / f u^{e}\right]=$ $\sum_{i=1}^{n-1}\left\langle a_{i} x^{i}\right\rangle\left[1, p_{i} x^{i} g f^{e-1} /(f u)^{e}\right] \in L_{d-1}$ by part (ii).

In particular, by the lemma, for any $\lambda \in \mathscr{F}$ and $h \in \mathscr{F}[x]$, both $\langle\lambda\rangle[1, h]$ and $\langle\lambda x\rangle[1, h x]$ lie in $L_{0}$. This will be used frequently.

Lemma 2.3. Suppose that $p$ is a monic irreducible polynomial of degree $d$. If $r \in F$ is a $v_{p}$-unit, and if $s$ is $v_{p}$-integral, then $\partial_{p}(\langle r\rangle[1, s])=0$. Consequently, if $\operatorname{deg} p=d$ and $\phi \in L_{d-1}$, we have $\partial_{p}(\phi)=0$.

Proof. Since $r$ is a $v_{p}$-unit we can write $r=r_{0}+p r^{\prime}$ with $0 \neq r_{0} \in \bar{F}_{p}$, where $r^{\prime}$ is $v_{p}$-integral. Next, in $F_{p}$ we can write $s\left(r_{0} / r\right)=s_{0}+p s^{\prime}$, where $s_{0} \in \bar{F}_{p}$ and $s^{\prime}$ is $v_{p}$-integral in $F_{p}$. Since $v_{p}\left(s\left(p r^{\prime} / r\right)\right)>0$ and $v_{p}\left(p s^{\prime}\right)>0$, we know that both $s\left(p r^{\prime} / r\right)$ and $p s^{\prime}$ lie in $\wp\left(F_{p}\right)$. Computing in $W_{q} F_{p}$ we find that

$$
\begin{aligned}
\langle r\rangle[1, s] & =\left\langle r_{0}+p r^{\prime}\right\rangle[1, s]=\left\langle r_{0}\right\rangle\left[1, s\left(r_{0} / r\right)\right]+\left\langle p r^{\prime}\right\rangle\left[1, s\left(p r^{\prime} / r\right)\right] \\
& =\left\langle r_{0}\right\rangle\left[1, s_{0}+p s^{\prime}\right]=\left\langle r_{0}\right\rangle\left[1, s_{0}\right] .
\end{aligned}
$$


Since each of $r_{0}$ and $s_{0}$ lie in $\bar{F}_{p}$ we see $\langle r\rangle[1, s] \in \operatorname{im}\left(W_{q} \bar{F}_{p} \rightarrow W_{q} F_{p}\right)$ and $\partial_{p}(\langle r\rangle[1, s])=0$ follows.

Now consider a generator $\langle f\rangle\left[1, h / u^{e}\right]$ for $L_{d-1}$. As $f, u \in \mathscr{F}[x]_{<d}$, we know that $h / u^{e}$ is $v_{p}$-integral and $f$ is a $v_{p}$-unit, so $\partial_{p}\left(\langle f\rangle\left[1, h / u^{e}\right]\right)=0$ and we are done in this case. Next consider a generator $\left[\lambda_{1} x^{i}, \lambda_{2} x^{j}\right]=\left\langle\lambda_{1} x^{i}\right\rangle\left[1, \lambda_{1} \lambda_{2} x^{i+j}\right]$ of $L_{0}$. If $p \neq x$ then $\lambda_{1} x^{i}$ is a $p$-adic unit and $\lambda_{1} \lambda_{2} x^{i+j}$ is $v_{p}$-integral. If $p=x$ and $i=0$, then again $\lambda_{1}$ is an $x$-adic unit and $\lambda_{1} \lambda_{2} x^{j}$ is $v_{p}$-integral. Otherwise if $p=x$ and $i>0$ we know that $v_{x}\left(\lambda_{1} \lambda_{2} x^{i+j}\right)>0$ and so $\lambda_{1} \lambda_{2} x^{i+j} \in \wp\left(F_{v_{x}}\right)$, giving $\left[\lambda_{1} x^{i}, \lambda_{2} x^{j}\right]=0 \in W_{q} F_{v_{x}}$. This proves the lemma.

Definition 2.4. Whenever $p$ is a monic irreducible polynomial we define $S_{p}$ to be the subgroup of $W_{q} \mathscr{F}(x)$ generated by all forms $\langle r\rangle\left[1, h / p^{s}\right]$, where $r \in \mathscr{F}$, $h \in \mathscr{F}_{[}[x]$, and $s \geq 0$. When $p=\frac{1}{x}$ we denote by $S_{\frac{1}{x}}$ the subgroup generated by all forms generated by $\langle r\rangle[1, h x]$, where $r \in \mathscr{F}$ and $h \in \mathscr{F}[x]$.

We observe that $W_{q} \mathscr{F}(x)=\bigcup_{d=0}^{\infty} L_{d}$. This is because the usual additive generators for $W_{q} \mathscr{F}(x)$ are included in some $L_{d}$ for large enough $d$. The next lemma describes several generating sets for the $L_{d}$.

Lemma 2.5. (i) $L_{d}=\sum_{p, \operatorname{deg} p \leq d}\left(S_{p}+\langle x\rangle S_{p}\right)$.

(ii) $W_{q} \mathscr{F}(x)=\sum_{p}\left(S_{p}+\langle x\rangle S_{p}\right)$.

(iii) When $d \geq 1, L_{d}$ is generated by $L_{d-1}$ and $S_{p} \cup\langle x\rangle S_{p}$, where deg $p=d$.

Proof. Part (i) follows from Lemma 2.2(ii) and partial fractions. Part (ii) follows from (i) since $W_{q} \mathscr{F}(x)=\bigcup_{d=0}^{\infty} L_{d}$. Part (iii) follows using partial fractions and (i).

We next establish a result from linear algebra needed to relate $\langle x\rangle S_{p}$ and $\langle p\rangle S_{p}$. For $p=x^{d}+p_{1} x^{d-1}+\cdots+p_{d-1} x+p_{d}$ we express each $p_{i}$ as $\sum_{K \in T} t^{K} p_{i, K}^{2}$, where $p_{i, J} \in \mathscr{F}$. For each $K \in T$ we define $P_{K} \in \overline{\mathscr{F}(x)_{p}}$ by

$$
P_{K}:= \begin{cases}\overline{t^{K}\left(p_{1, K}^{2} x^{d-1}+p_{3, K}^{2} x^{d-3}+\cdots+p_{d-1, K}^{2} x\right)} & \text { when } d \text { is even, } \\ \overline{t^{K}\left(x^{d}+p_{2, K}^{2} x^{d-2}+\cdots+p_{d-1, K}^{2} x\right)} & \text { when } d \text { is odd. }\end{cases}
$$

Next let $M$ be the $2^{n} \times 2^{n}$-matrix with entries indexed by the group $T$ and with $(I, J)$-th entry $P_{I+J}$. We show that $M$ is invertible:

Lemma 2.6. Assume $T$ is an elementary abelian 2-group with $2^{n}$ elements and $P_{K}$ are elements of a field of characteristic 2 indexed by $K \in T$. Suppose that $M$ is the $\left(2^{n} \times 2^{n}\right)$-matrix with $(I, J)$-th entry $P_{I+J}$. If $\sum_{K \in T} P_{K} \neq 0$, then $M$ is invertible.

Proof. Let Perm $T$ denote the set of permutations of $T$. We know that

$$
\operatorname{det} M=\sum_{\sigma \in \operatorname{Perm} T}\left(\prod_{\tau \in T} P_{\tau+\sigma(\tau)}\right)
$$


For each $s \in T$ we define $\sigma_{s} \in \operatorname{Perm} T$ by $\sigma_{s}(\tau)=\tau+s$. In this case $\tau+\sigma_{s}(\tau)=s$ for all $\tau$ and we have $\prod_{\tau \in T} P_{\tau+\sigma_{s}(\tau)}=P_{s}^{2^{n}}$.

Now let $T$ act on Perm $T$ via $\sigma^{\epsilon}(\tau)=\sigma(\tau+\epsilon)+\epsilon$ for all $\epsilon \in T$. (That this is an action is readily checked using the fact that $T$ is abelian.) If $\sigma^{\epsilon}=\sigma$ for all $\epsilon \in T$, then $\sigma(\epsilon)=\sigma^{\epsilon}(\epsilon)=\sigma(\epsilon+\epsilon)+\epsilon=\sigma(0)+\epsilon$ for all $\epsilon$, and we see that $\sigma=\sigma_{\sigma(0)}$ in this case. In particular, if $\sigma \neq \sigma_{s}$ for some $s \in T$ then the orbit of $\sigma$ under $T$ has more than one element. We next note that for any $\sigma \in \operatorname{Perm} T$ and $\epsilon \in T$ we have

$$
\prod_{\tau \in T} P_{\tau+\sigma^{\epsilon}(\tau)}=\prod_{\tau \in T} P_{\tau+\sigma(\tau+\epsilon)+\epsilon}=\prod_{(\tau+\epsilon) \in T} P_{(\tau+\epsilon)+\sigma(\tau+\epsilon)}=\prod_{\tau \in T} P_{\tau+\sigma(\tau)},
$$

and consequently for any $\sigma$ different from the $\sigma_{s}$ we have

$$
\sum_{\theta \in \operatorname{Orbit}(\sigma)}\left(\prod_{\tau \in T} P_{\tau+\theta(\tau)}\right)=\operatorname{card}(\operatorname{Orbit}(\sigma)) \prod_{\tau \in T} P_{\tau+\sigma^{\epsilon}(\tau)}=0,
$$

since $\operatorname{card}(\operatorname{Orbit}(\sigma))$ is a proper power of 2. Decomposing the sum in the determinant over the orbits in Perm $T$ shows that

$$
\operatorname{det} M=\sum_{K \in T} P_{K}^{2^{n}}=\left(\sum_{K \in T} P_{K}\right)^{2^{n}} .
$$

If $M$ fails to be invertible, we have $\operatorname{det} M=0$, which implies $\sum_{K \in T} P_{K}=0$, contrary to our hypothesis. The lemma is proved.

Corollary 2.7. If $p$ is irreducible and separable and if the $P_{K}$ are defined as in (4), then $M$ is invertible as a matrix over $\mathscr{F}(p):=\mathscr{F}[x] /(p)$.

Proof. Suppose that det $M=0$. Then $\sum_{K \in T} P_{K}=0$. Each $P_{K}$ lies in $\overline{t^{K} x \mathscr{F}(x)_{p}^{2}}$, and since the $t_{1}, t_{2}, \ldots, t_{n}$ remain 2-independent in $\overline{\mathscr{F}}(x)_{p}$ (because $p$ is separable), we see that each $P_{K}=0$. Now, since $1, x, x^{2}, \ldots x^{d-1}$ are linearly independent over $\mathscr{F}_{F}$, we find for all $K$ that each $p_{i, K}$ vanishes, where $i$ is odd when $d$ is even and even when $d$ is odd. So the same follows for the $p_{i}$. The first case contradicts the separability of $p$ and the second case contradicts the irreducibility of $p$.

We are now able to apply Corollary 2.7 and relate $S_{p},\langle x\rangle S_{p}$ and $\langle p\rangle S_{p}$. Whenever $p$ is not separable we choose $i$ so that $t_{i} \in \mathscr{F}(p)^{2}\left(t_{1}, \ldots, t_{i-1}\right)$ and then we denote by $\tilde{S}_{p}$ the subgroup of $S_{p}+\langle x\rangle S_{p}$ generated by the elements $\left\langle t^{I}\right\rangle\left[1, h / p^{e}\right]$, where $t^{I}$ is a product of $t_{1}, t_{2}, \ldots t_{j-1}, t_{j+1}, \ldots, t_{n}, x$.

Proposition 2.8. (i) For all $p$, we have $S_{p}+\langle p\rangle S_{p} \subseteq S_{p}+\langle x\rangle S_{p}$.

(ii) If $p$ is separable, $S_{p}+L_{0}+\langle x\rangle S_{p}=S_{p}+L_{0}+\langle p\rangle S_{p}$.

(iii) If $p$ is not separable, $S_{p}+L_{0}+\langle x\rangle S_{p}=\tilde{S}_{p}+L_{0}+\langle p\rangle \tilde{S}_{p}$. 
Proof. Part (i) essentially follows from Lemma 2.2(ii), but we give a direct calculation here because it is necessary for part (ii). Consider $\langle p\rangle\left[1, h / p^{s}\right] \in\langle p\rangle S_{p}$ and apply the fundamental relation in $W_{q}$ to obtain

$$
\langle p\rangle\left[1, \frac{h}{p^{s}}\right]=\sum_{j=0}^{d}\left\langle p_{j} x^{d-j}\right\rangle\left[1, \frac{p_{j} x^{d-j} h}{p^{s+1}}\right] .
$$

Whenever $d-j$ is even, we have

$$
\left\langle p_{j} x^{d-j}\right\rangle\left[1, \frac{p_{j} x^{d-j} h}{p^{s+1}}\right]=\left\langle p_{j}\right\rangle\left[1, \frac{p_{j} x^{d-j} h}{p^{s+1}}\right] \in S_{p},
$$

from which we find, modulo $S_{p}$,

$$
\begin{aligned}
\langle p\rangle\left[1, \frac{h}{p^{s}}\right] & \equiv \sum_{d-j \text { odd }}\left\langle p_{j} x^{d-j}\right\rangle\left[1, \frac{p_{j} x^{d-j} h}{p^{s+1}}\right] \equiv \sum_{d-j \text { odd }}\left(\sum_{K \in T}\left\langle t^{K} x\right\rangle\left[1, \frac{t^{K} p_{j, K}^{2} x^{d-j} h}{p^{s+1}}\right]\right) \\
& \equiv \sum_{K \in T}\left\langle t^{K} x\right\rangle\left[1, \frac{t^{K}\left(\sum_{d-j \text { odd }} p_{j, K}^{2} x^{d-j}\right) h}{p^{s+1}}\right] \equiv \sum_{K \in T}\left\langle t^{K} x\right\rangle\left[1, \frac{P_{K} h}{p^{s+1}}\right],
\end{aligned}
$$

where the second equivalence uses $p_{j}=\sum_{K \in T} t^{K} p_{j, K}^{2}$, the third changes order of summation, and the fourth uses the definition of the $P_{K}$. Part (i) follows from this.

For (ii) we consider the problem of reversing this process when $p$ is separable. Namely, we must express an element $\langle x\rangle\left[1, g / p^{s+1}\right] \in W_{q} \mathscr{F}(x)$ as a sum, modulo $S_{p}+L_{0}$, of elements of the form $\sum_{J}\left\langle t^{J} p\right\rangle\left[1, h_{J} / p^{s}\right]$. For this we denote by $S_{p, s}$ the subgroup of $S_{p}$ generated by the generators of $S_{p}$, where the exponent of $p$ doesn't exceed $s$. We can then proceed by backwards induction on $s$ and calculate in $\overline{F(x)_{v_{p}}}$. Multiplying the equivalence in the previous paragraph by $\left\langle t^{J}\right\rangle$ gives a system of $2^{n}$ such equivalences, one for each $J \in T$ :

$$
\left\langle t^{J} p\right\rangle\left[1, \frac{h_{J}}{p^{s}}\right] \equiv \sum_{K \in T}\left\langle t^{K+J} x\right\rangle\left[1, \frac{P_{K} h_{J}}{p^{s+1}}\right]\left(\bmod S_{p}\right) .
$$

Taking the sum gives, again modulo $S_{p}$,

$$
\sum_{J}\left\langle t^{J} p\right\rangle\left[1, \frac{h_{J}}{p^{s}}\right] \equiv \sum_{J} \sum_{K \in T}\left\langle t^{K+J} x\right\rangle\left[1, \frac{P_{K} h_{J}}{p^{s+1}}\right] \equiv \sum_{L}\left\langle t^{L} x\right\rangle\left[1, \frac{\sum_{J} P_{L+J} h_{J}}{p^{s+1}}\right],
$$

where in the second sum the variable $L$ is introduced to collect terms with like $K+J$. Since we want the latter sum to equal $\langle x\rangle\left[1, g / p^{s+1}\right]$, we obtain for the $h_{J}$ the equations

$$
\sum_{J} P_{J} h_{J}=g \text { and } \sum_{J} P_{L+J} h_{J}=0 \text { when } L \neq O
$$


in $\overline{\mathscr{F}(x)_{p}}$. In matrix form this system is

$$
M \cdot\left(h_{J}\right)=\left(\begin{array}{llll}
g & 0 & \cdots & 0
\end{array}\right)^{T},
$$

where $\left(h_{J}\right)$ means the column with $2^{n}$ entries aligned with corresponding entries of $M$, whose $(I, J)$-th entry is $P_{I+J}$. Since $M$ is invertible over $\overline{\mathscr{F}(x)_{p}}$ by Corollary 2.7, we can find elements $h_{J} \in \mathscr{F}[x]_{<\operatorname{deg} p}$ such that

$$
\langle x\rangle\left[1, \frac{g}{p^{s+1}}\right] \equiv \sum_{J}\left\langle t^{J} p\right\rangle\left[1, \frac{h_{J}}{p^{s}}\right] \quad\left(\bmod S_{p}+\langle x\rangle S_{p, s}\right) .
$$

By backwards induction on $s$ we can reduce to $s=0$. When $s=0$, the error terms are sums $\sum_{J}\left\langle t^{J} p\right\rangle\left[1, h_{J} p\right]$ lying in $L_{0}$ by Lemma 2.2(i). The result in (ii) follows.

For part (iii) we write $p=\sum_{j} p_{j} x^{d-j}$ and note that since $p$ is not separable, each $d-j$ is even, and we have a 2 -dependence between $t_{1}, t_{2}, \ldots t_{n-1}, t_{n}, p$. Reordering $t_{1}, t_{2}, \ldots, t_{n}$ we can assume that $t_{n} \in \mathscr{F}(x)^{2}\left(t_{1}, t_{2}, \ldots, t_{n-1}, p\right)$. This relabeling guarantees that $t_{1}, t_{2}, \ldots t_{n-1}, x, p$ is a basis for $\mathscr{F}(x)$ as well as $\mathscr{F}(x)_{p}$. We express each $p_{j}$ as $p_{0, j}+p_{1, j} t_{n}$, where $p_{i, j} \in \mathscr{F}(x)^{2}\left(t_{1}, t_{2}, \ldots, t_{n-1}\right)$, and further express each $p_{1, j}$ as $\sum_{J} t^{J} p_{1, j, J}^{2}$; here each $J_{n}$ vanishes. Then we can form

$$
\tilde{P}_{K}=t^{K}\left(p_{1,0, K}^{2} x^{d}+p_{1,2, K}^{2} x^{d-2}+\cdots+p_{1, d, K}^{2}\right)
$$

and note that $\sum_{K} \tilde{P}_{K}=\partial p / \partial t_{n} \neq 0 \in \mathscr{F}^{2}\left(t_{1}, t_{2}, \ldots, t_{n-1}\right)\left[x^{2}\right]$.

We can write

$$
\langle p\rangle\left[1, \frac{h}{p^{e}}\right]=\sum_{i}\left\langle p_{i}\right\rangle\left[1, \frac{p_{i} x^{d-i} h}{p^{e+1}}\right]=\sum_{i}\left\langle p_{0, i}+p_{1, i} t_{n}\right\rangle\left[1, \frac{p_{i} x^{d-i} h}{p^{e+1}}\right],
$$

and so, modulo $\tilde{S}_{p}$,

$$
\begin{aligned}
\langle p\rangle\left[1, \frac{h}{p^{e}}\right] & \equiv \sum_{i}\left\langle p_{1, i} t_{n}\right\rangle\left[1, \frac{p_{1, i} t_{n} x^{d-i} h}{p^{e+1}}\right] \\
& \equiv \sum_{i, K}\left\langle t^{K} t_{n}\right\rangle\left[1, \frac{t^{K} p_{1, i, K}^{2} t_{n} x^{d-i} h}{p^{e+1}}\right] \equiv \sum_{K}\left\langle t^{K} t_{n}\right\rangle\left[1, \frac{\tilde{P}_{K} t_{n} h}{p^{e+1}}\right] .
\end{aligned}
$$

What we must do is reverse this process and solve, modulo $\tilde{S}_{p}+L_{0}$, the congruence

$$
\begin{aligned}
\left\langle t_{n}\right\rangle\left[1, \frac{g}{p^{e+1}}\right] & \equiv \sum_{L}\left\langle t^{L} p\right\rangle\left[1, \frac{h_{L}}{p^{e}}\right] \equiv \sum_{K, L}\left\langle t^{L+K} t_{n}\right\rangle\left[1, \frac{\tilde{P}_{K} t_{n} h_{L}}{p^{e+1}}\right] \\
& \equiv \sum_{J}\left\langle t^{J} t_{n}\right\rangle\left[1, \frac{\sum_{L} \tilde{P}_{J+L} t_{n} h_{L}}{p^{e+1}}\right],
\end{aligned}
$$


for appropriate polynomials $h_{L}$. As in part (ii) it suffices to reduce the exponent $e$ by 1 . This system is equivalent to solving the system in $2^{n-1}$ variables in $\mathscr{F}(p)$, $g=\sum_{L} \tilde{P}_{L} t_{n} h_{L}$ and $0=\sum_{L} \tilde{P}_{K+L} t_{n} h_{L}$, where $K \neq O$. This can be written in matrix form as

$$
\tilde{M} \cdot\left(h_{L}\right)=\left(\begin{array}{llll}
g & 0 & \cdots & 0
\end{array}\right)^{T},
$$

for the $h_{L} \in \mathscr{F}(p)$. Here $\tilde{M}$ is the matrix with $(K, L)$-th entry $\tilde{P}_{K+L} t_{n} \in \mathscr{F}(p)$. However, we have noted that $\sum_{K} \tilde{P}_{K} \neq 0$, so the invertibility of $\tilde{M}$ follows from Lemma 2.6. This gives what is needed.

\section{The maps $\partial_{p}$ and their splitting}

From now on, unless stated otherwise, $p$ denotes either a monic irreducible polynomial in $\mathscr{F}[x]$ or $\frac{1}{x}$. Then $v_{p}$ denotes the associated valuation and we continue to use $F_{p}$ to denote the completion of $F=\mathscr{F}(x)$ at $v_{p}$. We use $F_{p}$ to denote the completion of $F=\mathscr{F}(x)$ at $v_{p}$. We continue to assume that $\mathscr{F}$ has a finite 2-basis $t_{1}, t_{2}, \ldots, t_{n}$. To apply the results from Section 1 we will need to specify a 2-basis for $\mathscr{F}(p):=\mathscr{F}[x] /(p)=\bar{F}_{p}$. So in this section we will have to be careful and keep track of separability conditions. We recall a well-known result, whose proof is embedded in the subsequent discussion, where we set up notation.

Lemma 3.1. A 2-basis for $\mathscr{F}$ is a 2-basis for $\mathscr{F}(p)$ if and only if $p$ is separable.

Since $t_{1}, t_{2}, \ldots, t_{n}$ is the fixed 2-basis for $\mathscr{F}_{\mathscr{F}}, t_{1}, t_{2}, \ldots, t_{n}, x$ is a 2 -basis for $F=\mathscr{F}(x)$. We express the monic irreducible $p \in \mathscr{F}[x]$ as

$$
p=\sum_{I \in T} t^{I}\left(p_{I}(x)\right)^{2}
$$

where $p_{I}(x) \in \mathscr{F}[x]$ and the multiindices $t^{I}$ refer to the 2-basis for $F$ (which includes $x$ ). Since $\bar{F}_{p} \cong \mathscr{F}_{[}[x] /(p)$, we find that $t_{1}, t_{2}, \ldots, t_{n}$ remain 2 -independent in $\bar{F}_{p}$ if and only if for some $I$ with $I_{n+1} \neq 0$ we also have $p_{I}(x) \neq 0$. But this happens if and only if $p(x)$ has a nonzero summand of odd degree, i.e., if and only if $p$ is separable. Now, when $p$ is separable, if $I_{n+1} \neq 0$ for some $p_{I}(x) \neq 0$ we take $t_{1}, t_{2}, \ldots, t_{n}$ as our 2-basis for $\bar{F}_{p} \subset F_{p}$ and then we can use $t_{1}, t_{2}, \ldots, t_{n}, p$ as our 2-basis for $F_{p}$.

Otherwise, when $p$ is not separable, we choose $j$ maximal with $I_{j} \neq 0$ for some $p_{I}(x) \neq 0$ and we note that in this case $t_{1}, t_{2}, \ldots, t_{j-1}, t_{j+1}, \ldots, t_{n}, \bar{x}$ is a 2-basis for $\bar{F}_{p}$. We then take $t_{1}, t_{2}, \ldots, t_{j-1}, t_{j+1}, \ldots, t_{n}, \bar{x}, p$ as our 2-basis for $F_{p}$. In this case we use the lifting to $t_{1}, t_{2}, \ldots, t_{j-1}, t_{j+1}, \ldots, t_{n-1}, x \in F$ to define the embedding $\bar{F}_{p} \hookrightarrow F_{p}$ needed to define $\partial_{p}: W_{q} F \rightarrow W_{1} F_{p}$.

We will need to keep track of products of elements of these various 2-bases. This will be accomplished by using three different notations for multiindex sets: 
- We use $\left\{t^{I} \mid I \in T\right\}$ to denote products of elements of the original 2-basis for $\mathscr{F}$.

- We use $\left\{t^{I} \mid I \in T_{p}\right\}$ to denote products of elements of the residue 2-basis of $\mathscr{F}(p)$ as described above (which vary depending upon whether $p$ is separable).

- We use $\left\{t^{I} \mid I \in \tilde{T}_{p}\right\}$ to denote products of elements of the local 2-basis of $F_{p}$ as described above.

In our study of $W_{q} F$ we will need to understand how $W_{q} F$ elements map to elements of $W_{1} F_{p}$, so we need to represent $W_{1} F_{p}$ classes in a special way. The next result is a consequence of Theorem 1.3. Whenever $s \in \mathscr{F}[x]$ we denote by $\bar{s} \in \mathscr{F}[x]_{<\operatorname{deg} p}$ the unique polynomial with $s \equiv \bar{s}(\bmod p)$. When applying Theorem 1.3, the Teichmüller lifting used is the one associated with the 2-basis $\tilde{T}_{p}$.

Theorem 3.2. (i) If $p$ is monic and irreducible, every class $\phi$ in $W_{1} F_{p}$ can be expressed uniquely as

$$
\phi=\psi \perp\langle p\rangle \phi_{2},
$$

where

$$
\psi=\sum_{I \in \tilde{T}_{p}}\left\langle t^{I}\right\rangle\left[1, \sum_{J, J+I>I} t^{J} u_{I, J}\right]
$$

$u_{I, J}=\sum_{r \geq 1} \overline{s_{I, J, r}^{2}} / p^{2 r}$ with $s_{I, J, r} \in \mathscr{F}[x]_{<\operatorname{deg} p}$ and $\phi_{2} \in W_{q} \bar{v} F_{p}$, and where $s_{I, J, r}$ and the Witt classes of $\psi$ in $W_{q} F_{p}$ and $\phi_{2}$ in $W_{q} \bar{v} F_{p}$ are uniquely determined by the class of $\phi$.

(ii) Every class $\phi$ in $W_{1} F_{\frac{1}{x}}$ can be expressed as

$$
\phi=\psi \perp\left\langle\frac{1}{x}\right\rangle \phi_{2},
$$

where $\psi=\sum_{I \in \tilde{T}_{1}}\left\langle t^{I}\right\rangle\left[1, \sum_{J, J+I>I} t^{J} u_{I, J}^{2}\right]$ with $u_{I, J} \in x \cdot \mathscr{F}[x]$ and $\phi_{2} \in W_{q} \mathscr{F}$, and where the Witt classes of $\psi$ in $W F_{p}$ and $\phi_{2}$ in $W_{q}$ F are uniquely determined by the class of $\phi$. In this expression we note that $\sum_{J, J+I>I} t^{J} u_{I, J}^{2} \in x \cdot \mathscr{F}[x]$.

Proof. In (i), since $\bar{F}_{p}=\mathscr{F}(p)$, applying valuation theory we conclude that every element $f \in \bar{F}_{p} \subset F_{p}$ can be expressed as $f_{1}+f_{2}$, where $f_{1} \in \mathscr{F}[x]_{<\operatorname{deg} p}, f_{2} \in F_{p}$, and $v_{p}\left(f_{2}\right)>0$. Consequently, any element $r \in R=\overline{\mathscr{F}(x)_{p}}\left[p^{-1}\right]$ can be expressed in the form $r=s_{1}+s_{2}$, where $s_{1} \in \mathscr{F}[x]_{<\operatorname{deg} p}^{2}\left[p^{-1}\right]$ and $v_{p}\left(s_{2}\right)>0$. Part (i) now follows, interpreting Theorem 1.3 in this setting and making the appropriate substitutions. Part (ii) is a direct consequence of Theorem 1.3, since there is no ambiguity about viewing the residue field as a subfield of $F_{\frac{1}{x}}$.

We next digress slightly and give a generator-relation structure for the Witt group. A very similar characterization was found by Arason [2003]. 
Theorem 3.3. For any field $F$ of characteristic 2 the Witt group $W_{q} F$ is isomorphic to $\left(F^{+} \otimes F^{+}\right) / \mathcal{W}$, where $W$ is the subgroup generated by

(i) $a \otimes b$ for $a b \in \wp(F)$,

(ii) $a \otimes b+b \otimes a$ for all $a, b \in F$, and

(iii) $a \otimes b+c \otimes a b / c$ whenever $c \in D_{F}[a, b]$.

Proof. We show that the epimorphism $F^{+} \otimes F^{+} \rightarrow W_{q} F$ defined by $a \otimes b \mapsto[a, b]$ has kernel $\mathcal{W}$. First we note that the generators of $\mathscr{W}$ map to trivial elements of $W_{q} F$. For type (i) generators, the Arf invariant of $a x^{2}+x y+b y^{2}$ is $a b$, and therefore $[a, b]=0 \in W_{q} F$ if and only if $a b \in \wp(F)$. Type (ii) generators vanish by the symmetry of $[a, b]$. For type (iii) generators, since $c \in D_{F}[a, b]$, we know the form $[a, b] \perp\langle c\rangle[1, a b]=\langle a, c\rangle[1, a b]$ is isotropic, hence hyperbolic. So $[a, b]=\langle c\rangle[1, a b]=[c, a b / c]$, which is what we want.

We next note that whenever $[a, b]=[c, d] \in W_{q} F$, and since $c \in D_{F}[a, b]$, we find $[a, b]=[c, a b / c]=[c, d]$, the later equality being equivalent to $[c, a b / c+d]=$ 0 . By our observations about (i) this is equivalent to $a b+c d \in \wp(F)$. This shows that all equalities $[a, b]=[c, d] \in W_{q} F$ are a consequence of multilinearity and the relations (i), (ii), and (iii).

Next, given an isomorphism $q=\left[a_{1}, b_{1}\right] \perp \psi \cong[c, d] \perp \chi$, we must show that it follows from the relations defining $\mathcal{W}$. For this we view each representation $q$ as having the same underlying vector space $V=F^{2 n}$ and we let $v, w \in V$ denote the first two symplectic basis elements in the second expression. Hence $q(v)=c$, $q(w)=d$ and the inner product $(v, w)_{q}$ equals 1 . We suppose $\psi=\left[a_{2}, b_{2}\right] \perp \cdots \perp$ $\left[a_{n}, b_{n}\right]$. If we view $v \in V$ according to the decomposition given by the first form, we can express $c$ as a sum $c_{1}+c_{2}+\cdots+c_{n}$, where $c_{i} \in D_{F}\left[a_{i}, b_{i}\right]$. Applying (iii) at each summand, we can write $\left[a_{1}, b_{1}\right] \perp\left[a_{2}, b_{2}\right] \perp \cdots \perp\left[a_{n}, b_{n}\right] \cong\left[c_{1}, e_{1}\right] \perp$ $\left[c_{2}, e_{2}\right] \perp \cdots \perp\left[c_{n}, e_{n}\right]$ for $e_{i}=a_{i} b_{i} / c_{i} \in F$. Using the bilinearity of the symbol $[$,$] , and since c=c_{1}+c_{2}+\cdots+c_{n}$, we have $\left[c_{1}, e_{1}\right] \perp\left[c_{2}, e_{2}\right] \perp \cdots \perp\left[c_{n}, e_{n}\right] \cong$ $\left[c, e_{1}\right] \perp\left[c_{2}, e_{1}+e_{2}\right] \perp \cdots \perp\left[c_{n}, e_{1}+e_{n}\right]$. If $v_{1}=v, w_{1}, v_{2}, w_{2}, \ldots, v_{n}, w_{n}$ is the symplectic basis corresponding to this new decomposition $q=\left[c, e_{1}\right] \perp$ $\left[c_{2}, e_{1}+e_{2}\right] \perp \cdots \perp\left[c_{n}, e_{1}+e_{n}\right]$, we can express $w$ as $z_{1}+z_{2}+\cdots+z_{n}$ with each $z_{i}$ a linear combination of $v_{i}$ and $w_{i}$. This means that if $d_{i}=q\left(z_{i}\right)$ for $1 \leq i \leq n$, then $d=q(w)=d_{1}+d_{2}+\cdots+d_{n}$. Since $(v, w)_{q}=1$ while $\left(v, z_{i}\right)_{q}=0$ for $2 \leq i \leq n$, we see that $\left(v, z_{1}\right)_{q}=1$. Since $\operatorname{span}\left(v, w_{1}\right)=\operatorname{span}\left(v, z_{1}\right)$, restricting our attention to this subspace we see that in fact $\left[c, e_{1}\right] \cong\left[c, d_{1}\right]$. We now apply relation (iii) to the other summands to obtain $\left[c_{i}, e_{1}+e_{i}\right] \cong\left[c_{i}^{\prime}, d_{i}\right]$ for $c_{i}^{\prime}=c_{i}\left(e_{1}+e_{i}\right) / d_{i} \in F$. Using bilinearity again we find $\left[c, e_{1}\right] \perp\left[c_{2}, e_{1}+e_{2}\right] \perp \cdots \perp\left[c_{n}, e_{1}+e_{n}\right] \cong\left[c, d_{1}\right] \perp$ $\left[c_{2}^{\prime}, d_{2}\right] \perp \cdots \perp\left[c_{n}^{\prime}, d_{n}\right] \cong[c, d] \perp\left[c+c_{2}^{\prime}, d_{2}\right] \perp \cdots \perp\left[c+c_{n}^{\prime}, d_{n}\right]$. Altogether, using only bilinearity and the rules (i), (ii), (iii), we have shown that our original $\left[a_{1}, b_{1}\right] \perp \psi$ is Witt equivalent to $[c, d] \perp \psi^{\prime}$ for some $\psi^{\prime}$. By Witt cancellation 
we now have $\psi^{\prime} \cong \chi$. By induction on $n$ we can reduce to the case $[a, b] \cong[c, d]$ already considered. This proves the theorem.

We now define the Milnor splittings.

Lemma and Definition 3.4. Suppose $p$ is a monic irreducible polynomial of degree $d \geq 1$ and that $\phi \in W_{1} F_{p}$ is of the form

$$
\phi=\psi \perp\langle p\rangle \phi_{2},
$$

where

$$
\psi=\sum_{I \in T_{p}}\left\langle t^{I}\right\rangle\left[1, \sum_{J, J+I>I} t^{J} r_{I, J}^{2}\right]
$$

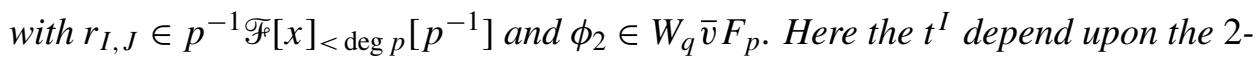
basis for $\mathscr{F}(x)_{p}$, which has last element $p$ and will include $x$ in the case where $p$ is not separable. We further write $\phi_{2}=\sum_{i}\left[r_{i}(\bar{x}), s_{i}(\bar{x})\right]$, where $\bar{x}$ denotes $x$ modulo $p(x)$ and the $r_{i}(x), s_{i}(x)$ lie in $\mathscr{F}[x]_{<\operatorname{deg}}$. Then the map $\tau_{p}$ defined by

$$
\tau_{p}(\phi)=\psi \perp\langle p\rangle\left(\sum_{i}\left[r_{i}(x), s_{i}(x)\right]\right)\left(\bmod L_{d-1}\right) \in L_{d} / L_{d-1}
$$

is a well defined homomorphism $\tau_{p}: W_{1} F_{p} \rightarrow L_{d} / L_{d-1}$.

Proof. According to Theorem 3.2(i) every class $\phi$ in $W_{1} F_{p}$ can be expressed as stated, and the Witt classes of $\psi \in W_{q} F_{p}$ and $\phi_{2} \in W_{q} \bar{F}_{p}$ are uniquely determined. Further, the Witt class of $\psi$ uniquely determines the $r_{I, J}$ as elements of

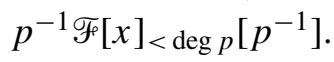

The expression of $\phi_{2}$ as $\sum_{i}\left[r_{i}(\bar{x}), s_{i}(\bar{x})\right]$ need not be unique so we suppose also that $\phi_{2}=\sum_{j}\left[u_{j}(\bar{x}), v_{j}(\bar{x})\right] \in W_{q} \mathscr{F}(p)$, where each $u_{j}(x)$ and $v_{j}(x)$ lies in $\mathscr{F}[x]_{<\operatorname{deg} p}$. By Theorem 3.3, using the biadditivity of the symbol [, ] we have the expansion

$$
\sum_{i}\left[r_{i}(\bar{x}), s_{i}(\bar{x})\right]+\sum_{j}\left[u_{j}(\bar{x}), v_{j}(\bar{x})\right]=\sum_{k}\left[a_{k}(\bar{x}), b_{k}(\bar{x})\right],
$$

where the latter sum is a sum of relations of the form given in Theorem 3.3(i), (ii) or (iii). Since we only used the biadditivity of $[$,$] in the expansion, we$ know that each $a_{k}(x), b_{k}(x) \in \mathscr{F}[x]_{<\operatorname{deg} p}$ and we also have $\sum_{i}\left[r_{i}(x), s_{i}(x)\right]+$ $\sum_{j}\left[u_{j}(x), v_{j}(x)\right]=\sum_{k}\left[a_{k}(x), b_{k}(x)\right] \in W_{q} \mathscr{F}(x)$. Checking for each of the types of relations given in Theorem 3.3 we will show that $\langle p\rangle$ times this sum lies in $L_{d-1}$.

Suppose first we have a summand $[a, b]$ with $a, b \in \mathscr{F}[x]_{<\operatorname{deg} p}$, where $\bar{a} \bar{b} \in$ $\wp(\mathscr{F}(p))$. Then we can write $a b=\wp(z)+p g$ in $\mathscr{F}[x]$ and we find that $[a, b]=$ $\langle a\rangle[1, a b]=\langle a\rangle[1, \wp(z)+p g]=\langle a\rangle[1, p g]$ in $W_{q} F$. By Lemma 2.2(iii) the form $\langle p\rangle\langle a\rangle[1, p g]$ lies in $L_{d-1}$ since $a \in \mathscr{F}[x]_{<\operatorname{deg} p}$. Next, any pair in the sum of the form $[a, b]+[b, a]$ is zero in $W_{q} F$ as well. Finally, suppose we have a pair in 
the sum $[a, b]+[c, d]$, where $\bar{c} \in D_{\mathscr{F}(p)}[\bar{a}, \bar{b}]$ and $\bar{d}=\overline{a b / c}$ where $a, b, c, d \in$ $\mathscr{F}[x]_{<\operatorname{deg} p}$. Then we can write $c=a r^{2}+r s+b s^{2}+p g$ and $a b=c d+p h$ in $\mathscr{F}[x]$ where $r, s \in \mathscr{F}[x]_{<\operatorname{deg} p}$ also. Since $\left(s / r+b s^{2} / r^{2}\right) b \in \wp(F)$, we obtain $\left[s / r+b s^{2} / r^{2}, b\right]=0$. Rewriting the expression for $c$, we find $a=c / r^{2}+s / r+$ $b s^{2} / r^{2}+p g / r^{2}$, so in $W_{q} F$ we have

$$
\begin{aligned}
{[a, b]+[c, d] } & =\left[c / r^{2}, b\right]+\left[s / r+b s^{2} / r^{2}, b\right]+\left[p g / r^{2}, b\right]+[c, d] \\
& =\langle c\rangle\left[1 / r^{2}, b c\right]+\left[p g / r^{2}, b\right]+\langle c\rangle[1, c d] \\
& =\langle c\rangle\left[1, b c / r^{2}\right]+\langle c\rangle[1, c d]+\left[p g / r^{2}, b\right] .
\end{aligned}
$$

Next, substituting $b c / r^{2}=a b+b s / r+b^{2} s^{2} / r^{2}+b p g / r^{2}$ and $c d=a b+p h$ we find

$$
\begin{aligned}
{[a, b]+[c, d] } & =\langle c\rangle\left[1, a b+b s / r+b^{2} s^{2} / r^{2}+p g b / r^{2}\right]+\langle c\rangle[1, a b+p h]+\left[p g / r^{2}, b\right] \\
& =\langle c\rangle\left[1, p g b / r^{2}+p h\right]+\left[p g / r^{2}, b\right],
\end{aligned}
$$

since $b s / r+b^{2} s^{2} / r^{2} \in \wp(F)$. Applying Lemma 2.2(iii) to each of these latter forms we find that $\langle p\rangle([a, b]+[c, d])$ lies in $L_{d-1}$. It follows that $\tau_{p}$ is well defined. It is clear from the defining formula that $\tau_{p}$ is additive in $\psi$, and the proof that the lift of $\phi_{2}$ is well defined shows that $\tau_{p}$ is additive in that term as well. Hence $\tau_{p}$ is a homomorphism.

Our goal is to prove the surjectivity of the Milnor splitting. This requires the information provided in Proposition 2.8 and is given next.

Theorem 3.5. The map $\bigoplus \tau_{p}: \bigoplus_{p, \operatorname{deg} p=d} W_{1} F(x)_{v_{p}} \rightarrow L_{d} / L_{d-1}$ is an isomorphism for $d \geq 1$.

Proof. It suffices to show the map is surjective, since for any $p$ the composite $W_{1} F(x)_{v_{p}} \rightarrow L_{d} / L_{d-1} \rightarrow W_{1} F(x)_{v_{p}}$ is the identity. (Here, the first map is $\tau_{p}$ and the second map is $\partial_{p}$, which vanishes on $L_{d-1}$ by Lemma 2.3.) By the definition of $\tau_{p}$, every element $\psi=\sum_{I \in T_{p}}\left\langle t^{I}\right\rangle\left[1, \sum_{J, J+I>I} t^{J} r_{I, J}^{2}\right]$ with $r_{I, J} \in$ $p^{-1} \mathscr{F}[x]_{<\operatorname{deg} p}\left[p^{-1}\right]$ lies in the image. When $p$ is separable, these elements generate $S_{p}+\langle p\rangle S_{p}$ so we have $S_{p}+\langle p\rangle S_{p} \subseteq \operatorname{im}\left(\tau_{p}\right)$, and when $p$ is not separable, these elements generate $\tilde{S}_{p}+\langle p\rangle \tilde{S}_{p}$ and we have $\tilde{S}_{p}+\langle p\rangle \tilde{S}_{p} \subseteq \operatorname{im}\left(\tau_{p}\right)$. Further, if $p$ is separable, then $S_{p}+\langle p\rangle S_{p}+L_{0}=S_{p}+\langle x\rangle S_{p}+L_{0}$ by Proposition 2.8(ii) and in case $p$ is not separable we have $\tilde{S}_{p}+\langle p\rangle \tilde{S}_{p}+L_{0}=S_{p}+\langle x\rangle S_{p}+L_{0}$ by Proposition 2.8(iii). However, $L_{d}$ is generated by $S_{p} \cup\langle x\rangle S_{p}$ for $p$ with $\operatorname{deg} p=d$ together with $L_{d-1}$ by Lemma 2.5. From this the theorem is proved.

The definition of $L_{0}$ combined with Theorem 3.2(ii) gives:

Theorem 3.6. There is an exact sequence

$$
0 \rightarrow W_{q} \mathscr{F} \rightarrow L_{0} \rightarrow W_{1} F_{\frac{1}{x}} \rightarrow W_{q} \mathscr{F} \rightarrow 0,
$$


where the first two maps are induced by inclusion and the last map is $\phi \mapsto \phi_{2}$, where $\phi_{2}$ is as given in Theorem 3.2(ii).

Proof. Since $L_{0}$ is generated by the forms $\left[\lambda_{1} x^{i}, \lambda_{2} x^{j}\right]=\left\langle\lambda_{1} x^{i}\right\rangle\left[1, \lambda_{1} \lambda_{2} x^{i+j}\right]$, applying the relations in $W_{q} F$ we see that every element $\phi \in L_{0}$ can be expressed as $\phi=\phi_{1} \perp \psi$, where $\phi_{1} \in W_{q} \mathscr{F}$ and $\psi=\sum_{I+J>I}\left\langle t^{I}\right\rangle\left[1, t^{J} r_{I, J}^{2}\right]$ with $r_{I, J} \in x$. F $[x]$. Moreover, the expression of $\psi$ as such a sum is unique, according to the local theory at the $v_{\frac{1}{x}}$-adic valuation as given in Theorem 3.2(ii). This means that the natural map from $L_{0}$ to $W_{1} F_{\frac{1}{x}}$ has kernel $W_{q} \mathscr{F}$ and cokernel the elements in $\left\langle\frac{1}{x}\right\rangle W_{q} \mathscr{F}$. The result follows.

\section{The transfer maps $s_{p}^{*}$}

We continue to use the 2-bases for $\mathscr{F}(p)$ and $\mathscr{F}(x)_{p}$ defined in the discussion following Lemma 3.1, as well as the notation $T, T_{p}$ and $\tilde{T}_{p}$. When $p$ fails to be separable and $I \in T_{p}$, we denote by $I_{x}$ the entry corresponding to the exponent of $x$ (so $x$ occurs in $t^{I}$ if and only if $I_{x}=1$.) In the next definition, we define subgroups $S_{p, r}$ of $S_{p}+\langle p\rangle S_{p}$, for each $r \geq 1$. We include $p=\frac{1}{x}$ in our list. We set $d=\operatorname{deg} p$ and $d=1$ when $p=\frac{1}{x}$. As in the last section, when considering elements of $S_{p}$ of the form $\left\langle t^{I}\right\rangle\left[1, h / p^{r}\right]$ for $h \in \mathscr{F}[x]$, we write $\bar{h} \in \mathscr{F}[x]_{<\operatorname{deg} p}$ for the unique element with $h \equiv \bar{h}(\bmod p)$.

Definition 4.1. (i) Suppose $p$ is separable or is $\frac{1}{x}$. We define $S_{p, r} \subset S_{p}+\langle p\rangle S_{p}$ as the subgroup generated by elements of two types: those of the form

$$
\left\langle t^{I}\right\rangle\left[1, t^{J} \overline{s_{I, J}^{2}} / p^{r}\right],
$$

where $I, J \in T=T_{p}, s_{I, J} \in \mathscr{F}[x]_{<d}$, and where $I+J>I$ whenever $r$ is even, and those of the form

$$
\left\langle t^{I} p\right\rangle\left[1, t^{J} \overline{s_{I, J}^{2}} / p^{r}\right],
$$

where $I, J \in T=T_{p}, s_{I, J} \in \mathscr{F}_{[}[x]_{<d}, r$ is even, and where $I+J>I$.

(ii) Suppose $p$ is not separable. We define $S_{p, r} \subset \tilde{S}_{p}+\langle p\rangle \tilde{S}_{p}$ as the subgroup generated by elements of two types: those of the form

$$
\left\langle t^{I}\right\rangle\left[1, t^{J} \overline{s_{I, J}^{2}} / p^{r}\right],
$$

where $I, J \in T=T_{p}, s_{I, J} \in \mathscr{F}[x]_{<d}$, and where $I+J>I$ whenever $r$ is even, and those of the form

$$
\left\langle t^{I} p\right\rangle\left[1, t^{J} \overline{s_{I, J}^{2}} / p^{r}\right],
$$

where $I, J \in T=T_{p}, s_{I, J} \in \mathscr{F}_{[}[x]_{<d}, r$ is even, and where $I+J>I$. 
(iii) Suppose $p$ is not separable. We define $S_{p, r}^{0} \subset S_{p, r}$ as the subgroup generated by elements of two types: those of the form

$$
\left\langle t^{I}\right\rangle\left[1, t^{J} \overline{s_{I, J}^{2}} / p^{r}\right],
$$

where $I_{x}=0, I, J \in T_{p}, s_{I, J} \in \mathscr{F}[x]_{<d}$, and where $I+J>I$ whenever $r$ is even, and those of the form

$$
\left\langle t^{I} p\right\rangle\left[1, t^{J} \overline{s_{I, J}^{2}} / p^{r}\right],
$$

where $I_{x}=0, I, J \in T_{p}, r$ is even, $s_{I, J} \in \mathscr{F}[x]_{<d}$, and where $I+J>I$.

(iv) We define $U_{p}:=\sum_{r \geq 1} S_{p, r}$ for all $p$ and $U_{p}^{0}:=\sum_{r \geq 1} S_{p, r}^{0}$ when $p$ is not separable.

The definitions in (i) and (ii) are formally the same, except that the $T_{p}$ 's differ according to whether $p$ is separable or not, which also accounts for using $S_{p}$ or $\tilde{S}_{p}$. In part (iii) the listed elements are a subcollection of those listed in (ii), and are precisely those without an $x$ in the $t^{I}$. The reason for the restrictions on $I, J, r$ in the definition will become clear in the proof of the next lemma, where we apply Theorem 3.2.

Lemma 4.2. (i) For each $p, S_{p}+\langle p\rangle S_{p} \subseteq U_{p}+L_{0}+\langle p\rangle L_{0}$.

(ii) The group

$$
U_{p}=\bigoplus_{r \geq 1} S_{p, r} \subset S_{p}+\langle p\rangle S_{p}
$$

is a direct sum.

(iii) Let the image of this group in $W_{1} F_{p}$ also be denoted by $U_{p}$. Then

$$
W_{1} F_{p} / U_{p} \cong\langle p\rangle \cdot W_{q} \mathscr{F}(p) .
$$

Thus every element in $W_{1} F_{p} / U_{p}$ can be represented by an element of $\langle p\rangle L_{0}$.

Proof. (i) This follows from the additive property of the symbol [1,a], expanding elements of $S_{p}$ into sums of $S_{p, r}$ elements one power of $p$ at a time, leaving an element of the form $[1, g]$ where $g \in \mathscr{F}[x]$. The last summands lie in $L_{0}$.

(ii) The summands from $\psi$ in Theorem 3.2 can be uniquely expressed as a sum of elements of the form

$$
\left\langle t^{I}\right\rangle\left[1, t^{J} \overline{s_{I, J, r}^{2}} / p^{2 r}\right],
$$

where $I, J \in \tilde{T}_{p}$ satisfy $I+J>I$ and $s_{I, J, r} \in \mathscr{F}[x]_{<\operatorname{deg} p}$. Given this, we claim that the generators identified in Definition 4.1 are equivalent to those required to apply Theorem 3.2. There are four cases, depending upon whether $I, J \in T_{p}$ or not. In case both $I, J \in T_{p}$, then the condition that $I+J>I$ is the same for $T_{p}$ elements as for $\tilde{T}_{p}$ elements, and this is recorded in Definition 4.1 in the first type where $r$ is even. In case $I \in T_{p}$ but $J \notin T_{p}$, then $I+J>I$ is automatic and this 
corresponds to the first type in Definition 4.1 where $r$ is odd. In case, $I \notin T_{p}$ but $J \in T_{p}$ then $I+J>I$ in $\tilde{T}_{p}$ is equivalent to $I^{\prime}+J>I^{\prime}$ in $T_{p}$ where $I^{\prime}$ is the same as $I$ except the $p$ component is deleted, and this is the case of the second type of generator in Definition 4.1. Finally, if both $I, J \notin T_{p}$, then $I+J>I$ is impossible as elements of $\tilde{T}_{p}$, and this case is ignored by Definition 4.1 . So we are ready to apply Theorem 3.2.

First, every sum of elements of $S_{p, r}$ can be represented as

$$
\sum_{r \geq 1} \sum_{I+J>I}\left\langle t^{I}\right\rangle\left[1, \frac{t^{J} \overline{s_{I, J, r}^{2}}}{p^{r}}\right]+\sum_{r \geq 2, \text { even }} \sum_{I+J>I}\left\langle t^{I} p\right\rangle\left[1, \frac{t^{J} \overline{s_{I, J, r}^{\prime 2}}}{p^{r}}\right],
$$

where $s_{I, J, r}, s_{I, J, r}^{\prime}$ lie in $\mathscr{F}[x]_{<d}$ and as elements of $T_{p}$ we have $I+J>I$ in each summand. These expressions can be rewritten as

$$
\sum_{I+J>I}\left\langle t^{I}\right\rangle\left[1, \sum_{r \geq 1} \frac{t^{J} \overline{s_{I, J, r}^{2}}}{p^{r}}\right]+\sum_{I+J>I}\left\langle t^{I} p\right\rangle\left[1, \sum_{r \geq 2, \text { even }} \frac{t^{J} \overline{s_{I, J, r}^{\prime 2}}}{p^{r}}\right] .
$$

Now, in Theorem 3.2 the 2-basis $\hat{T}_{p}$ is used, which is in this case consists of the elements of $T_{p}$ and $p \cdot T_{p}$. So each of the sums in the additive slots of the last expression,

$$
\sum_{r \geq 1} \frac{t^{J} \overline{s_{I, J, r}^{2}}}{p^{r}} \text { and } \sum_{r \geq 2, \text { even }} \frac{t^{J} \overline{s_{I, J, r}^{\prime 2}}}{p^{r}}
$$

correspond uniquely to elements listed as $t^{J} s_{I, J, r}^{2}$ in the statement of Theorem 3.2(i). The directness of the sum is follows. The statement in (iii) is also a consequence of Theorem 3.2, proving the lemma.

We are now able to define the transfer maps $s_{p}^{*}$.

Definition 4.3. For $\psi \in W_{1} F_{p}$ we define $\theta_{p}(\psi) \in W_{q} \mathscr{F}(p)$ to be the unique element of $W_{q} \bar{v} F_{p}$ for which $\psi \equiv\langle p\rangle \theta_{p}(\psi)\left(\bmod U_{p}\right)$. We denote by $t_{p}^{*}: \mathscr{F}(p) \rightarrow \mathscr{F}$ the Scharlau transfer associated to the linear functional $t_{p}$ for which $t_{p}\left(x^{d-1}\right)=1$ but $t_{p}\left(x^{i}\right)=0$ when $0 \leq i<d-1$. Finally, whenever $f \in \mathscr{F}[x]$ is a polynomial we denote by $f_{c}$ its constant term.

(i) Suppose $p$ is separable or $\frac{1}{x}$. Then we define $s_{p}^{*}(\psi):=t_{p}^{*}\left(\theta_{p}(\psi)\right) \in W_{q} \mathscr{F}$.

(ii) If $p$ is not separable we express $\psi-\theta_{p}(\psi)$ modulo $U_{p}^{0}$ as

$$
\sum_{I_{x}=1, r}\left\langle t^{I}\right\rangle\left[1, \overline{t^{J} s_{I, J, r}^{2}}\right]+\sum_{I_{x}=1, r}\left\langle\left(t^{I}\right) p\right\rangle\left[1, \frac{\overline{t^{J} s_{I, J, r}^{2}}}{p^{r}}\right] .
$$


Then we define $s_{p}^{*}(\psi) \in \in W_{q} \mathscr{F}$ by

$s_{p}^{*}(\psi):=t_{p}^{*}\left(\theta_{p}(\psi)\right)+\sum_{I_{x}=1, r}\left\langle t^{I} / x\right\rangle\left[1, \frac{\left(t^{J} s_{I, J, r}^{2}\right)_{c}}{p_{c}^{r}}\right]+\sum_{I_{x}=1, r}\left\langle t^{I} p_{c} / x\right\rangle\left[1, \frac{\left(t^{J} s_{I, J, r}^{2}\right)_{c}}{p_{c}^{r}}\right]$.

(Note that $t^{I} / x \in \mathscr{F}$ since $I_{x}=1$.)

To prove reciprocity, we must check it for each $\langle p\rangle L_{0}$ and each $U_{p}$. This will be done in the next two sections.

\section{The reciprocity law for $L_{0}+\langle p\rangle L_{0}$}

In this section we prove the reciprocity law in a critical special case. We assume $p=x^{d}+p_{1} x^{d-1}+\cdots+p_{d} \in \mathscr{F}[x]$ is a monic irreducible polynomial of degree $d=2 e$ when $d$ is even, and of degree $d=2 e+1$ when $d$ is odd. Since we will be calculating in both $\mathscr{F}[x]$ and $\mathscr{F}[x] /(p)$ we will use $x$ to represent the variable in $\mathscr{F}[x]$ as well as its residue in $\mathscr{F}[x] /(p)$, since no confusion will arise. For $h \in\{0,1\}$, $\lambda_{1}, \lambda_{2} \in \mathscr{F}_{F}$, and $k \geq 0$ we will compute the transfer $t_{p}^{*}\left(\left[\lambda_{1} x^{h}, \lambda_{2} x^{k}\right]\right)=\phi \in W_{q} \mathscr{F}_{F}$ described in Definition 4.3. We note that in case $h^{\prime}>1$ we can write $h^{\prime}=h+2 h_{0}$ for $h \in\{0,1\}$ and as $\left[\lambda_{1} x^{h^{\prime}}, \lambda_{2} x^{k}\right]=\left\langle\lambda_{1} x^{h^{\prime}}\right\rangle\left[1, \lambda_{1} \lambda_{2} x^{k+h^{\prime}}\right]=\left\langle\lambda_{1} x^{h}\right\rangle\left[1, \lambda_{1} \lambda_{2} x^{k+h^{\prime}}\right]=$ $\left[\lambda_{1} x^{h}, \lambda_{2} x^{k+h^{\prime}-h}\right]$ we see there is no loss of generality in our restriction on $h$.

For $0 \leq j \leq e$ we define the polynomials $f_{j}=x^{e+j}+p_{1} x^{e+j-1}+\cdots+p_{2 j} x^{e-j}$ and $g_{j}=x^{e+j}+p_{1} x^{e+j-1}+\cdots+p_{2 j+1} x^{e-j-1}$. Thus $g_{j}=f_{j}+p_{2 j+1} x^{e-j-1}$. We next define $\gamma_{i} \in \mathscr{F}$ by expressing $x^{d+i-1}=\gamma_{i} x^{d-1}+G_{i} \in \mathscr{F}[x] /(p)$ where $G_{i} \in \mathscr{F}[x]$ is a polynomial of degree at most $d-2$. Note that this means that $t_{p}\left(x^{d+i-1}\right)=\gamma_{i}$. Clearly, $\gamma_{0}=1$, and using the equation $x^{d}=p_{1} x^{d-1}+\cdots+p_{d} \in \mathscr{F}(p):=\mathscr{F}[x] /(p)$ we see that $\gamma_{1}=p_{1}$ and by induction for $i \geq 1$,

$$
\begin{aligned}
x^{d+i-1} & =p_{1} x^{d+i-2}+p_{2} x^{d+i-3}+\cdots+p_{d} x^{i-1} \\
& =\left(p_{1} \gamma_{i-1} p_{2} \gamma_{i-2}+\cdots+p_{i} \gamma_{0}\right) x^{d-1}+p_{1} G_{i-1} p_{2} G_{i-2}+\cdots+p_{i} G_{0} .
\end{aligned}
$$

This shows that in general the $\gamma_{i}$ satisfy the recurrence relation

$$
\gamma_{i}=\gamma_{i-1} p_{1}+\gamma_{i-2} p_{2}+\cdots \gamma_{0} p_{i} \quad \text { for } i \geq 1 \text {. }
$$

In fact, this recurrence relation is the same as the relation that guarantees that, as power series, $\left(1+p_{1} X+p_{2} X^{2}+\cdots\right)\left(1+\gamma_{1} X+\gamma_{2} X^{2}+\cdots\right)=1 \in \mathscr{F}((X))$, and which we use below in proving Lemma 5.3.

Lemma 5.1. (i) Suppose $d=2 e$. We have $t_{p}\left(f_{0}^{2}\right)=\gamma_{1}=p_{1}$ and $t_{p}\left(x f_{0}^{2}\right)=\gamma_{2}=$ $p_{1}^{2}+p_{2}$. For all $j$ with $1 \leq j \leq e$ we have $t_{p}\left(f_{j}^{2}\right)=p_{2 j+1}, t_{p}\left(x f_{j}^{2}\right)=p_{2 j+2}$. For all $k \geq 0$ we have $t_{p}\left(x^{k} g_{0}^{2}\right)=\gamma_{k+1}+p_{1}^{2} \gamma_{k-1}$. 
(ii) Suppose $d=2 e+1$. We have $t_{p}\left(f_{0}^{2}\right)=\gamma_{0}=1$ and $t_{p}\left(x f_{0}^{2}\right)=\gamma_{1}=p_{1}$. For all $j$ with $1 \leq j \leq e$ we have $t_{p}\left(f_{j}^{2}\right)=p_{2 j}, t_{p}\left(x f_{j}^{2}\right)=p_{2 j+1}$. For all $k \geq 0$ we have and $t_{p}\left(x^{k} g_{0}^{2}\right)=\gamma_{k}+p_{1}^{2} \gamma_{k-2}$.

Proof. Since $f_{0}=x^{e}$ we have $t\left(x^{h} f_{0}^{2}\right)=t\left(x^{h+2 e}\right)$ so when $d=2 e$ we find $t\left(x^{h} f_{0}^{2}\right)=\gamma_{h+1}$ and when $d=2 e+1$ we find $t\left(x^{h} f_{0}^{2}\right)=\gamma_{h}$ as required. When $j>0$ and $d=2 e$ we have in $\mathscr{F}(p)$

$$
\begin{aligned}
f_{j}^{2} & =\left(x^{e+j}+p_{1} x^{e+j-1}+\cdots+p_{2 j} x^{e-j}\right)^{2} \\
& =\left(x^{2 e}+p_{1} x^{2 e-1}+\cdots+p_{2 j} x^{2 e-2 j}\right)\left(x^{2 j}+p_{1} x^{2 j-1}+\cdots+p_{2 j}\right) \\
& =\left(p_{2 j+1} x^{d-2 j-1}+\cdots+p_{d}\right)\left(x^{2 j}+p_{1} x^{2 j-1}+\cdots+p_{2 j}\right) \\
& =p_{2 j+1} x^{d-1}+\left(p_{2 j+1} p_{1}+p_{2 j+2}\right) x^{d-2}+\cdots+p_{d} p_{2 j} .
\end{aligned}
$$

We find that $t_{p}\left(f_{j}^{2}\right)=t_{p}\left(p_{2 j+1} x^{d-1}\right)=p_{2 j+1} \gamma_{0}=p_{2 j+1}$ and that $t_{p}\left(x f_{j}^{2}\right)=$ $t_{p}\left(p_{2 j+1} x^{d}+\left(p_{2 j+1} p_{1}+p_{2 j+2}\right) x^{d-1}\right)=p_{2 j+1} \gamma_{1}+\left(p_{2 j+1} p_{1}+p_{2 j+2}\right) \gamma_{0}=p_{2 j+2}$ as $\gamma_{0}=1$ and $\gamma_{1}=p_{1}$.

When $j>0$ and $d=2 e+1$ we have

$$
\begin{aligned}
& f_{j}^{2}=\left(x^{e+j}+p_{1} x^{e+j-1}+\cdots+p_{2 j} x^{e-j}\right)^{2} \\
&=\left(x^{2 e+1}+p_{1} x^{2 e}+\cdots+p_{2 j} x^{2 e-2 j+1}\right)\left(x^{2 j-1}+p_{1} x^{2 j-2}+\cdots+p_{2 j-1}\right) \\
& \quad+\left(x^{2 e}+p_{1} x^{2 e-1}+\cdots+p_{2 j} x^{2 e-2 j}\right) p_{2 j} \\
&=\left(p_{2 j+1} x^{d-2 j-1}+\cdots+p_{d}\right)\left(x^{2 j-1}+p_{1} x^{2 j-2}+\cdots+p_{2 j-1}\right) \\
& \quad+\left(x^{2 e}+p_{1} x^{2 e-1}+\cdots+p_{2 j} x^{2 e-2 j}\right) p_{2 j} \\
&=p_{2 j} x^{d-1}+\left(p_{2 j+1}+p_{1} p_{2 j}\right) x^{d-2}+\cdots+p_{d} p_{2 j-1} .
\end{aligned}
$$

So we find $t_{p}\left(f_{j}^{2}\right)=p_{2 j}$ and $t_{p}\left(x f_{j}^{2}\right)=p_{2 j} \gamma_{1}+\left(p_{2 j} p_{1}+p_{2 j+1}\right) \gamma_{0}=p_{2 j+1}$, as $\gamma_{0}=1$ and $\gamma_{1}=p_{1}$.

Finally, as $g_{0}=x^{e}+p_{1} x^{e-1}$, we find that $x^{k} g_{0}^{2}=x^{2 e+k}+p_{1}^{2} x^{2 e+k-2}$ and therefore $t_{p}\left(x^{k} g_{0}^{2}\right)=\gamma_{k+1}+p_{1}^{2} \gamma_{k-1}$ when $d=2 e$ and $t_{p}\left(x^{k} g_{0}^{2}\right)=\gamma_{k}+p_{1}^{2} \gamma_{k-2}$ when $d=2 e+1$. This proves the lemma.

The next lemma calculates the transfers.

Lemma 5.2. Let $\lambda_{1}, \lambda_{2} \in \mathscr{F}, h \in\{0,1\}$, and $k \geq 0$. When $d=2 e$, the transfer is given by

$$
\begin{array}{r}
t_{p}^{*}\left(\left[\lambda_{1} x^{h}, \lambda_{2} x^{k}\right]\right)=\left[\lambda_{1} h, \lambda_{2}\left(\gamma_{k+1}+p_{1}^{2} \gamma_{k-1}\right)\right]+\sum_{i=1}^{e-1}\left[\lambda_{1} p_{2(e-i)+h+1}, \lambda_{2} \gamma_{k+2 i-d-1}\right] \\
+\left[\lambda_{1} \gamma_{h+1}, \lambda_{2} \gamma_{k-1}\right]
\end{array}
$$


and when $d=2 e+1$ by

$$
t_{p}^{*}\left(\left[\lambda_{1} x^{h}, \lambda_{2} x^{k}\right]\right)=\sum_{i=0}^{e}\left[\lambda_{1} p_{2(e-i)+h}, \lambda_{2} \gamma_{k+2 i-d+1}\right] .
$$

Proof. We first give a symplectic basis for $t_{p}^{*}\left(\left[\lambda_{1} x^{h}, \lambda_{2} x^{k}\right]\right)$. For $(r, s) \in \mathscr{F}[x] /(p) \times$ $\mathscr{F}[x] /(p)$, applying the quadratic form we have

$$
t_{p}^{*}\left(\left[\lambda_{1} x^{h}, \lambda_{2} x^{j}\right]\right)(r, s)=t_{p}\left(\lambda_{1} x^{h} r^{2}+r s+\lambda_{2} x^{j} s^{2}\right) .
$$

If $d=2 e$ is even we consider the basis

$$
\begin{aligned}
& \left\{(1,0),\left(0, g_{e-1}\right) ;(x, 0),\left(0, g_{e-2}\right) ; \ldots ;\left(x^{e-1}, 0\right),\left(0, g_{0}\right) ;\right. \\
& \left.\left(f_{e-1}, 0\right),(0,1) ;\left(f_{e-2}, 0\right),(0, x) ; \ldots ;\left(f_{0}, 0\right),\left(0, x^{e-1}\right)\right\} .
\end{aligned}
$$

If $d=2 e+1$ is odd we consider the basis

$$
\begin{aligned}
& \left\{(1,0),\left(0, g_{e}\right) ;(x, 0),\left(0, g_{e-1}\right) ; \ldots ;\left(x^{e-1}, 0\right),\left(0, g_{1}\right) ;\right. \\
& \left.\left(f_{e}, 0\right),(0,1) ;\left(f_{e-1}, 0\right),(0, x) ; \ldots ;\left(0, x^{e}\right),\left(f_{0}, 0\right)\right\} .
\end{aligned}
$$

In each case we claim the basis is symplectic. When $d=2 e$, since each polynomial $x^{i-1} f_{e-i}$ and $x^{i-1} g_{e-i}$ is monic of degree $d-1$ when $1 \leq i \leq e$ we see that the inner products $\left(\left(x^{i-1}, 0\right),\left(0, g_{e-i}\right)\right)$ and $\left(\left(0, x^{i-1}\right),\left(f_{e-i}, 0\right)\right)$ equal 1. Likewise, when $d=2 e+1$ each of $\left(\left(x^{i}, 0\right),\left(0, g_{e-i}\right)\right)$ and $\left(\left(0, x^{i}\right),\left(f_{e-i}, 0\right)\right)$ equal 1. Next, whenever $i+j \leq d-2$ we have $\left(\left(x^{i}, 0\right),\left(0, x^{j}\right)\right)=0$, showing that such pairs are always orthogonal. Since the product $x^{i} f_{e-j}$ has degree $i+2 e-j$, when $d=2 e$ and $i<j$ we have $t_{p}\left(x^{i-1} f_{e-j}\right)=0$, and when $d=2 e+1$ with $i<j$ we have $t_{p}\left(x^{i} f_{e-j}\right)=0$. For $i>j$, when $d=2 e$, we have

$$
\begin{aligned}
x^{i-1} f_{e-j} & =x^{i-1}\left(x^{2 e-j}+\cdots+p_{2(e-j)} x^{j}\right) \\
& =\left(x^{d}+\cdots+p_{2(e-j)} x^{2 j}\right) x^{i-j-1}=\left(p_{2(e-j)+1} x^{2 j-1}+\cdots+p_{d}\right) x^{i-j-1},
\end{aligned}
$$

this last having degree $i+j-2<d-1$. For $i>j$, when $d=2 e+1$, we have $x^{i} f_{e-j}=x^{i-1}\left(x^{2 e-j}+\cdots+p_{2(e-j)} x^{j}\right)=\left(x^{d}+\cdots+p_{2(e-j)} x^{2 j}\right) x^{i-j-1}=$ $\left(p_{2(e-j)+1} x^{2 j-1}+\cdots+p_{d}\right) x^{i-j-1}$, this last having degree $i+j-2<d-1$. So we see that the inner products $\left(\left(f_{e-j}, 0\right),\left(0, x^{i-1}\right)\right)$ vanish whenever $i \neq j$ and $d=2 e$, while $\left(\left(f_{e-j}, 0\right),\left(0, x^{i}\right)\right)$ vanish whenever $i \neq j$ and $d=2 e+1$. This also shows that when $i \neq j$ the inner product $\left(\left(0, g_{e-j}\right),\left(x^{i-1}, 0\right)\right)$ vanishes as well when $d=2 e$ :

$$
\begin{aligned}
\left(\left(0, g_{e-j}\right),\left(x^{i-1}, 0\right)\right) & =\left(\left(0, f_{e-j}+p_{2(e-j)+1} x^{j-1}\right),\left(x^{i-1}, 0\right)\right) \\
& =t_{p}\left(p_{2(e-j)+1} x^{i+j-2}\right)=0 .
\end{aligned}
$$

Similarly $\left(\left(0, g_{e-j}\right),\left(x^{i}, 0\right)\right)=0$ when $d=2 e+1$. 
So to check the required orthogonality we must calculate $t_{p}\left(g_{i} f_{j}\right)$ where $0 \leq$ $i, j \leq e-1$ and $j=e$ as well as when $d=2 e+1$. When $d=2 e$ and $j>i$ or $d=2 e+1$ and $j>i+1$ we calculate

$$
\begin{aligned}
t_{p}\left(g_{i} f_{j}\right) & =t_{p}\left(\left(x^{e+i}+\cdots+p_{2 i+1} x^{e-i-1}\right)\left(x^{e+j}+\cdots+p_{2 j} x^{e-j}\right)\right) \\
& =t_{p}\left(\left(x^{2 i+1}+\cdots+p_{2 i+1}\right)\left(x^{2 e+j-i-1}+\cdots+p_{2 j} x^{2 e-j-i-1}\right)\right) \\
& =t_{p}\left(\left(x^{2 i+1}+\cdots+p_{2 i+1}\right)\left(p_{2 j+1} x^{2 e-j-i-2}+\cdots+p_{d} x^{j-i-1}\right)\right)=0,
\end{aligned}
$$

because the latter polynomial has degree $2 e+i-j-1<d-1$. When $d=2 e$ and $j \leq i$ or $d=2 e+1$ and $i \leq j+1$ we calculate

$$
\begin{aligned}
t_{p}\left(g_{i} f_{j}\right) & =t_{p}\left(\left(x^{e+i}+\cdots+p_{2 i+1} x^{e-i-1}\right) x^{e-j}\left(x^{2 j}+\cdots+p_{2 j}\right)\right) \\
& =t_{p}\left(\left(x^{2 e+i-j}+\cdots+p_{2 i+1} x^{2 e-i-j-1}\right)\left(x^{2 j}+\cdots+p_{2 j}\right)\right) \\
& =t_{p}\left(\left(p_{2 i+2} x^{2 e-i-j-2}+\cdots+p_{d} x^{i-j}\right)\left(x^{2 j}+\cdots+p_{2 j}\right)\right)=0,
\end{aligned}
$$

because the latter polynomial has degree $2 e-i+j-2<d-1$. This shows that the inner products $\left(\left(f_{i}, 0\right),\left(0, g_{j}\right)\right)=0$ and therefore both bases listed are symplectic.

We are now able to compute the transfer $t_{p}^{*}\left(\left[\lambda_{1} x^{h}, \lambda_{2} x^{k}\right]\right)$, where $h \in\{0,1\}$. When $d=2 e$, since $h \leq 1$ the vectors $\left(x^{i}, 0\right)$ are isotropic as long as $0 \leq i<e-1$. So we can apply the previous lemma and we only need to use the portion of the symplectic basis that involves $g_{0}$ and the $f_{j}$. We find

$$
\begin{aligned}
t_{p}^{*}\left(\left[\lambda_{1} x^{h}, \lambda_{2} x^{k}\right]\right)= & {\left[t_{p}\left(\lambda_{1} x^{h} x^{2(e-1)}\right), t_{p}\left(\lambda_{2} x^{k} g_{0}^{2}\right)\right]+\sum_{i=1}^{e}\left[t_{p}\left(\lambda_{1} x^{h} f_{e-i}^{2}\right), t_{p}\left(\lambda_{2} x^{k} x^{2(i-1)}\right)\right] } \\
= & {\left[\lambda_{1} h, \lambda_{2}\left(\gamma_{k+1}+p_{1}^{2} \gamma_{k-1}\right)\right] } \\
& \quad+\sum_{i=1}^{e-1}\left[\lambda_{1} p_{2(e-i)+h+1}, \lambda_{2} \gamma_{k+2 i-d-1}\right]+\left[\lambda_{1} \gamma_{h+1}, \lambda_{2} \gamma_{k-1}\right],
\end{aligned}
$$

where in this last summand we have used $t\left(x^{h} f_{0}^{2}\right)=\gamma_{h+1}$. When $d=2 e+1$ we note that, since $h \leq 1$, each of the vectors $\left(x^{i}, 0\right)$, where $0 \leq i \leq e-1$, is isotropic. Hence we need only consider the part of the symplectic basis involving the $f_{j}$ and we find

$$
\begin{aligned}
t_{p}^{*}\left(\left[\lambda_{1} x^{h}, \lambda_{2} x^{k}\right]\right) & =\sum_{i=0}^{e}\left[t_{p}\left(\lambda_{1} x^{h} f_{e-i}^{2}\right), t_{p}\left(\lambda_{2} x^{k} x^{2 i}\right)\right] \\
& =\sum_{i=0}^{e}\left[\lambda_{1} p_{2(e-i)+h}, \lambda_{2} \gamma_{k+2 i-d+1}\right],
\end{aligned}
$$

where in the latter sum we have used $\gamma_{h}=p_{h}$ for $h=0,1$ when $i=e$. This proves the lemma.

We next have to compute $\partial_{\frac{1}{x}}\left(\langle p\rangle\left[\lambda_{1} x^{h}, \lambda_{2} x^{k}\right]\right)=\partial_{\frac{1}{x}}\left(\left\langle p x^{h}\right\rangle\left[\lambda_{1}, \lambda_{2} x^{h+k}\right]\right)$. There are four cases, depending upon the parity of $h$ and $d$ : 


\section{Lemma 5.3.}

$$
\begin{aligned}
s_{\frac{1}{x}}^{*}\left(\partial_{\frac{1}{x}}\left(\langle p\rangle\left[\lambda_{1}, \lambda_{2} x^{k}\right]\right)\right) & = \begin{cases}\sum_{j=0}^{e-1}\left[\lambda_{1} p_{2 j+1}, \lambda_{2} \gamma_{k-2 j-1}\right] & \text { if } d \text { is even, } \\
\sum_{j=0}^{e}\left[\lambda_{1} p_{2 j}, \lambda_{2} \gamma_{k-2 j}\right] & \text { if } d \text { is odd } ;\end{cases} \\
s_{\frac{1}{x}}^{*}\left(\partial_{\frac{1}{x}}\left(\langle p x\rangle\left[\lambda_{1}, \lambda_{2} x^{k+1}\right]\right)\right) & = \begin{cases}\sum_{j=0}^{e}\left[\lambda_{1} p_{2 j}, \lambda_{2} \gamma_{k-2 j+1}\right] & \text { if } d \text { is even, }, \\
\sum_{j=0}^{e}\left[\lambda_{1} p_{2 j+1}, \lambda_{2} \gamma_{k-2 j}\right] & \text { if } d \text { is odd. },\end{cases}
\end{aligned}
$$

Proof. As $p=x^{d}+p_{1} x^{d-1}+\cdots+p_{d}$ we are able to express $\left\langle p x^{h}\right\rangle\left[1, x^{h+k}\right]$ as

$$
\begin{aligned}
\left\langle x^{d+h}\right\rangle\left[1, x^{h+k+d} p^{-1}\right]+\left\langle p_{1} x^{d+h-1}\right\rangle\left[1, p_{1} x^{h+k+d-1} p^{-1}\right] & \\
& +\cdots+\left\langle p_{d} x^{h}\right\rangle\left[1, p_{d} x^{h+k} p^{-1}\right] .
\end{aligned}
$$

Since $p=x^{d}\left(1+p_{1} x^{-1}+p_{2} x^{-2}+\cdots p_{d} x^{-d}\right)$, inside the completion $\mathscr{F}(x)_{\frac{1}{x}}$ we can write $p^{-1}=x^{-d}\left(1+\gamma_{1} x^{-1}+\gamma_{2} x^{-2}+\cdots\right)$. When $d+h-i$ is even we have $s_{\frac{1}{x}}^{*}\left(\left\langle p_{i} x^{d+h-i}\right\rangle\left[\lambda_{1}, \lambda_{2} p_{i} x^{h+k+d-i} p^{-1}\right]\right)=0$ and when $d+h-i$ is odd we have

$$
\begin{aligned}
s_{\frac{1}{x}}^{*}\left(\langle p _ { i } x ^ { d + h - i } \rangle \left[\lambda_{1}, \lambda_{2} p_{i} x^{h+k+d-i}\right.\right. & \left.\left.p^{-1}\right]\right) \\
& =s_{\frac{1}{x}}^{*}\left(\left\langle p_{i} x^{-1}\right\rangle\left[\lambda_{1}, \lambda_{2} p_{i} x^{h+k-i}\left(1+\gamma_{1} x^{-1}+\cdots\right)\right]\right) \\
& =\left\langle p_{i}\right\rangle\left[\lambda_{1}, \lambda_{2} p_{i} \gamma_{h+k-i}\right] .
\end{aligned}
$$

So when $h=0$ and $d$ is even we have

$$
\begin{aligned}
s_{\frac{1}{x}}^{*}\left(\partial_{\frac{1}{x}}\left(\langle p\rangle\left[\lambda_{1}, \lambda_{2} x^{k}\right]\right)\right)= & \left\langle p_{1}\right\rangle\left[\lambda_{1}, \lambda_{2} p_{1} \gamma_{k-1}\right]+\left\langle p_{3}\right\rangle\left[\lambda_{1}, \lambda_{2} p_{3} \gamma_{k-3}\right] \\
& +\cdots+\left\langle p_{d-1}\right\rangle\left[\lambda_{1}, \lambda_{2} p\right. \\
= & \sum_{j=0}^{e-1}\left[\lambda_{1} p_{2 j+1}, \lambda_{2} \gamma_{k-2 j-1}\right] .
\end{aligned}
$$

Similarly, if $h=1$ and $d$ is even we find

$$
\begin{aligned}
s_{\frac{1}{x}}^{*}\left(\partial_{\frac{1}{x}}\left(\langle p x\rangle\left[\lambda_{1}, \lambda_{2} x^{k+1}\right]\right)\right)= & \left\langle p_{0}\right\rangle\left[\lambda_{1}, \lambda_{2} p_{0} \gamma_{k+1}\right]+\left\langle p_{2}\right\rangle\left[\lambda_{1}, \lambda_{2} p_{2} \gamma_{k-1}\right] \\
& +\cdots+\left\langle p_{d}\right\rangle\left[\lambda_{1}, \lambda_{2} p_{d} \gamma_{k-d+1}\right] \\
= & \sum_{j=0}^{e}\left[\lambda_{1} p_{2 j}, \lambda_{2} \gamma_{k-2 j+1}\right] .
\end{aligned}
$$

Next, if $h=0$ and $d$ is odd,

$$
\begin{aligned}
s_{\frac{1}{x}}^{*}\left(\partial_{\frac{1}{x}}\left(\langle p\rangle\left[\lambda_{1}, \lambda_{2} x^{k+1}\right]\right)\right)= & \left\langle p_{0}\right\rangle\left[\lambda_{1}, \lambda_{2} p_{0} \gamma_{k}\right]+\left\langle p_{2}\right\rangle\left[\lambda_{1}, \lambda_{2} p_{2} \gamma_{k-2}\right] \\
& +\cdots+\left\langle p_{2 e}\right\rangle\left[\lambda_{1}, \lambda_{2} p_{2 e} \gamma_{k-2 e}\right] \\
= & \sum_{j=0}^{e}\left[\lambda_{1} p_{2 j}, \lambda_{2} \gamma_{k-2 j}\right] .
\end{aligned}
$$


Finally, if $h=1$ and $d$ is odd,

$$
\begin{aligned}
s_{\frac{1}{x}}^{*}\left(\partial_{\frac{1}{x}}\left(\langle p x\rangle\left[\lambda_{1}, \lambda_{2} x^{k}\right]\right)\right)= & \left\langle p_{1}\right\rangle\left[\lambda_{1}, \lambda_{2} p_{1} \gamma_{k}\right]+\left\langle p_{3}\right\rangle\left[\lambda_{1}, \lambda_{2} p_{3} \gamma_{k-2}\right] \\
& +\cdots+\left\langle p_{d-1}\right\rangle\left[\lambda_{1}, \lambda_{2} p_{d-1} \gamma_{k-d+1}\right] \\
= & \sum_{j=0}^{e-1}\left[\lambda_{1} p_{2 j+1}, \lambda_{2} \gamma_{k-2 j}\right] .
\end{aligned}
$$

Theorem 5.4. The reciprocity law $\sum_{q} s_{q}^{*}\left(\partial_{q}(\phi)\right)=0$ holds for all $\phi \in L_{0}+\langle p\rangle L_{0}$. Proof. We first consider a generator $\phi=\left[\lambda_{1} x^{i}, \lambda_{2} x^{j}\right]$ of $L_{0}$. By Lemma 2.3, $\partial_{p}(\phi)$ vanishes for all $p \neq \frac{1}{x}$. When $p=\frac{1}{x}$, Theorem 3.6 shows that $s_{\frac{1}{x}}^{*}(\phi)=0$. So the reciprocity law holds for elements of $L_{0}$. We next note that for any generator $\phi=\langle p\rangle\left[\lambda_{1} x^{h}, \lambda_{2} x^{k}\right]$ of $\langle p\rangle L_{0}$ we have $s_{q}^{*}\left(\partial_{q}(\phi)\right)=0$ as long as $q \neq p, \frac{1}{x}$. So we must check that $s_{p}^{*}\left(\partial_{p}(\phi)\right)=s_{\frac{1}{x}}^{*}\left(\partial_{\frac{1}{x}}(\phi)\right)$ for all such generators.

When $d=2 e$ and $h=0$, we have by Lemma 5.2

$$
t_{p}^{*}\left(\left[\lambda_{1}, \lambda_{2} x^{k}\right]\right)=\sum_{i=1}^{e-1}\left[\lambda_{1} p_{2(e-i)+1}, \lambda_{2} \gamma_{k+2 i-d-1}\right]+\left[\lambda_{1} \gamma_{1}, \lambda_{2} \gamma_{k-1}\right] .
$$

Also in this case by Lemma 5.3 we have

$$
s_{\frac{1}{x}}^{*}\left(\partial_{\frac{1}{x}}\left(\langle p\rangle\left[\lambda_{1}, \lambda_{2} x^{k}\right]\right)\right)=\sum_{j=0}^{e-1}\left[\lambda_{1} p_{2 j+1}, \lambda_{2} \gamma_{k-2 j-1}\right],
$$

But $p_{1}=\gamma_{1}$; therefore the terms in these sums match exactly, which shows that $s_{p}^{*}\left(\partial_{p}\left(\langle p\rangle\left[\lambda_{1}, \lambda_{2} x^{k}\right]\right)\right)=t_{p}^{*}\left(\left[\lambda_{1}, \lambda_{2} x^{k}\right]\right)=s_{\frac{1}{x}}^{*}\left(\partial_{\frac{1}{x}}\left(\langle p\rangle\left[\lambda_{1}, \lambda_{2} x^{k}\right]\right)\right)$ in this case.

When $d=2 e$ and $h=1$, we have by Lemma 5.2

$$
\begin{array}{r}
t_{p}^{*}\left(\left[\lambda_{1} x, \lambda_{2} x^{k}\right]\right)=\left[\lambda_{1}, \lambda_{2}\left(\gamma_{k+1}+p_{1}^{2} \gamma_{k-1}\right)\right]+\sum_{i=1}^{e-1}\left[\lambda_{1} p_{2(e-i)+2}, \lambda_{2} \gamma_{k+2 i-d-1}\right] \\
+\left[\lambda_{1} \gamma_{2}, \lambda_{2} \gamma_{k-1}\right] .
\end{array}
$$

Also in this case by Lemma 5.3 we have

$$
s_{\frac{1}{x}}^{*}\left(\partial_{\frac{1}{x}}\left(\langle p\rangle\left[\lambda_{1} x, \lambda_{2} x^{k}\right]\right)\right)=\sum_{j=0}^{e}\left[\lambda_{1} p_{2 j}, \lambda_{2} \gamma_{k-2 j+1}\right] .
$$

These two expressions will be equal provided we can show

$$
\left[\lambda_{1}, \lambda_{2}\left(\gamma_{k+1}+p_{1}^{2} \gamma_{k-1}\right)\right]+\left[\lambda_{1} \gamma_{2}, \lambda_{2} \gamma_{k-1}\right]=\left[\lambda_{1}, \lambda_{2} \gamma_{k+1}\right]+\left[\lambda_{1} p_{2}, \lambda_{2} \gamma_{k-1}\right]
$$

since the summands $\sum_{i=1}^{e-1}\left[\lambda_{1} p_{2(e-i)+2}, \lambda_{2} \gamma_{k+2 i-d-1}\right]$ correspond exactly to the summands $\sum_{j=2}^{e}\left[\lambda_{1} p_{2 j}, \lambda_{2} \gamma_{k-2 j+1}\right]$. So we need that

$$
\left[\lambda_{1}, \lambda_{2} p_{1}^{2} \gamma_{k-1}\right]+\left[\lambda_{1} \gamma_{2}, \lambda_{2} \gamma_{k-1}\right]=\left[\lambda_{1} p_{2}, \lambda_{2} \gamma_{k-1}\right]
$$


which follows because $\left[\lambda_{1}, \lambda_{2} p_{1}^{2} \gamma_{k-1}\right]=\left\langle p_{1}^{2}\right\rangle \cdot\left[\lambda_{1} p_{1}^{2}, \lambda_{2} \gamma_{k-1}\right]$ and $\gamma_{2}=p_{1}^{2}+p_{2}$.

When $d=2 e+1$ and $h=0$, we have by Lemma 5.2

$$
t_{p}^{*}\left(\left[\lambda_{1}, \lambda_{2} x^{k}\right]\right)=\sum_{i=0}^{e}\left[\lambda_{1} p_{2(e-i)}, \lambda_{2} \gamma_{k+2 i-d+1}\right] .
$$

Also in this case by Lemma 5.3 we have

$$
s_{\frac{1}{x}}^{*}\left(\partial_{\frac{1}{x}}\left(\langle p\rangle\left[\lambda_{1}, \lambda_{2} x^{k}\right]\right)\right)=\sum_{j=0}^{e}\left[\lambda_{1} p_{2 j}, \lambda_{2} \gamma_{k-2 j}\right],
$$

which shows that $t_{p}^{*}\left(\left[\lambda_{1}, \lambda_{2} x^{k}\right]\right)=s_{\frac{1}{x}}^{*}\left(\partial_{\frac{1}{x}}\left(\langle p\rangle\left[\lambda_{1}, \lambda_{2} x^{k}\right]\right)\right)$, since the summations are the same apart from indexing.

When $d=2 e+1$ and $h=1$, we have by Lemma 5.2

$$
t_{p}^{*}\left(\left[\lambda_{1} x, \lambda_{2} x^{k}\right]\right)=\sum_{i=0}^{e}\left[\lambda_{1} p_{2(e-i)+1}, \lambda_{2} \gamma_{k+2 i-d+1}\right] .
$$

Also in this case by Lemma 5.3 we have

$$
s_{\frac{1}{x}}^{*}\left(\partial_{\frac{1}{x}}\left(\langle p\rangle\left[\lambda_{1} x, \lambda_{2} x^{k}\right]\right)\right)=\sum_{j=0}^{e}\left[\lambda_{1} p_{2 j+1}, \lambda_{2} \gamma_{k-2 j}\right]
$$

which shows that $t_{p}^{*}\left(\left[\lambda_{1}, \lambda_{2} x^{k}\right]\right)=s_{\frac{1}{x}}^{*}\left(\partial_{\frac{1}{x}}\left(\langle p\rangle\left[\lambda_{1}, \lambda_{2} x^{k}\right]\right)\right)$ again since the summations are the same. This gives the reciprocity law for $\langle p\rangle L_{0}$.

\section{The reciprocity law and the analogue of Milnor's theorem}

We next turn to the reciprocity law for $W_{q} \mathscr{F}(x)$.

Theorem 6.1. The composite $W_{q} F \stackrel{\bigoplus \partial_{p}}{\longrightarrow} \bigoplus_{p, \frac{1}{x}} W_{1} F_{p} \stackrel{\bigoplus s_{p}^{*}}{\longrightarrow} W_{q} \mathscr{F}$ is zero.

Proof. According to Lemma 2.5(ii), $W_{q} \mathscr{F}(x)=\sum_{p}\left(S_{p}+\langle x\rangle S_{p}\right)$. So it suffices to check the composite vanishes on $S_{p}+\langle x\rangle S_{p}$ for each $p$. In case $p=\frac{1}{x}$ then $S_{\frac{1}{x}}+\langle x\rangle S_{\frac{1}{x}} \subset L_{0}+\left\langle\frac{1}{x}\right\rangle L_{0}=L_{0}+\langle x\rangle L_{0}$. Since the reciprocity law holds for $L_{0}+\langle x\rangle L_{0}$ by Theorem 5.4, we can assume that $p$ is monic and irreducible. By Lemma 4.2, we know that $S_{p}+\langle x\rangle S_{p} \subset U_{p}+L_{0}+\langle p\rangle L_{0}$. By Theorem 5.4 we know the composite vanishes on $L_{0}+\langle p\rangle L_{0}$. Since $U_{p}=\sum_{r} S_{p, r}$, therefore, it suffices to verify the composite vanishes on each generator of $S_{p, r}$. If $q$ is not one of $p, x, \frac{1}{x}$, we know by Lemma 2.3 that $\partial_{p}$ vanishes on $S_{p, r}$, so we only need to worry about those three primes. 
We consider first the generators of $S_{p, r}$ of the form $\phi=\left\langle t^{I}\right\rangle\left[1, h / p^{r}\right] \in S_{p}$ or $\phi=\left\langle t^{I} p\right\rangle\left[1, h / p^{r}\right] \in\langle p\rangle S_{p}$ where $\operatorname{deg} h<\operatorname{deg} p$ and $r \geq 1$. Since $t^{I} \in \mathscr{F}$ we can assume $I=0$ by Frobenius reciprocity. If $p \neq x$ then $p$ is both an $x$-unit and a $\frac{1}{x}$-adic unit. This means $v_{x}\left(h / p^{r}\right) \geq 0$ and since $\operatorname{deg} h<\operatorname{deg} p$ we must have $v_{\frac{1}{x}}\left(h / p^{r}\right)>0$. So by Lemma 2.3 we find that $\partial_{x}(\phi)=\partial_{\frac{1}{x}}(\phi)=0$ in these cases. However by definition we know that $s_{p}^{*}\left(\partial_{p}(\phi)\right)=0$ for these particular generators of $U_{p}$ so reciprocity is established in these cases. In case $p=x$ then $v_{\frac{1}{x}}(h / x)>0$ so $\partial_{x}(\phi)=\partial_{\frac{1}{x}}(\phi)=0$ in this case as well. In case $p$ is separable we know that all generators for $U_{p}$ are of the form just considered so we are done when $p$ is separable. When $p$ is not separable, the generators just considered are the generators in $U_{p}^{0}$, so we are done in that case as well.

Finally, when $p$ is not separable, we must consider generators of $U_{p}$ that don't lie in $U_{p}^{0}$. These have the form $\left\langle t^{I} x\right\rangle\left[1, h / p^{r}\right] \in \tilde{S}_{p}$ or $\left\langle t^{I} p x\right\rangle\left[1, h / p^{r}\right] \in\langle p\rangle \tilde{S}_{p}$ where $\operatorname{deg} h<\operatorname{deg} p$ and $r \geq 1$. Again, since $t^{I} \in \mathscr{F}$ we can by Frobenius reciprocity assume $I=0$. In these cases we have by Definition 4.3(ii) that $s_{x}^{*}\left(\partial_{x}\left(\langle x\rangle\left[1, h / p^{r}\right]\right)\right)=$ $\left[1, h_{c} / p_{c}^{r}\right]$, and $s_{x}^{*}\left(\partial_{x}\left(\langle p x\rangle\left[1, h / p^{r}\right]\right)\right)=\left\langle p_{c}\right\rangle\left[1, h_{c} / p_{c}^{r}\right]$. Since $v_{\frac{1}{x}}\left(h / p^{r}\right)>0$ we have $s_{\frac{1}{x}}^{*}\left(\partial_{\frac{1}{x}}\left(\langle x\rangle\left[1, h / p^{r}\right]\right)\right)=0$, and $s_{\frac{1}{x}}^{*}\left(\partial_{\frac{1}{x}}\left(\langle p x\rangle\left[1, h / p^{r}\right]\right)\right)=0$. By the definition of $s_{p}^{*}$, we know that $s_{p}^{*}\left(\partial_{p}\left(\langle x\rangle\left[1, h / p^{r}\right]\right)=\left[1, h_{c} / p_{c}^{r}\right]\right.$ and $s_{p}^{*}\left(\partial_{p}\left(\langle p x\rangle\left[1, h / p^{r}\right]\right)=\right.$ $\left\langle p_{0}\right\rangle\left[1, h_{c} / p_{c}^{r}\right]$, giving the reciprocity law in this case.

Putting everything together gives the main result of the paper.

Theorem 6.2 (Analogue of the Milnor-Scharlau Sequence). Suppose that $\mathscr{F}_{F}$ is a field of characteristic 2 and $F=\mathscr{F}(x)$ is a rational function field in one variable over F. There exists a compatible collection of second residue and transfer maps that fit into an exact sequence

$$
0 \longrightarrow W_{q} \mathscr{F} \longrightarrow W_{q} F \stackrel{\bigoplus \partial_{p}}{\longrightarrow} \bigoplus_{p, \frac{1}{x}} W_{1} F_{p} \stackrel{\bigoplus s_{p}^{*}}{\longrightarrow} W_{q} \mathscr{F} \longrightarrow 0
$$

where the direct sum is taken over discrete valuations on $F$.

Proof. Everything completed previously applies when $\mathscr{F}$ has a finite 2-basis. In that case Theorem 3.5 shows that

$$
W_{q} F / L_{0} \cong \bigoplus_{d \geq 1} L_{d} / L_{d-1} \rightarrow \bigoplus_{p} W_{1} F_{p}
$$

is an isomorphism. Theorem 3.6 shows that

$$
0 \rightarrow W_{q} \mathscr{F} \rightarrow L_{0} \rightarrow W_{1} F_{\frac{1}{x}} \rightarrow W_{q} \mathscr{F} \rightarrow 0
$$

is an exact sequence. Theorem 6.1 shows we can patch the two sequences together and obtain the result. When $\mathscr{F}$ does not have a finite 2-basis, the result follows from the finite 2-basis case because any element in any group in the sequence lies 
in the same sequence defined for a finitely generated subfield of $\mathscr{F}$. This proves the theorem.

\title{
References
}

[Arason 1979] J. K. Arason, "Wittring und Galoiscohomologie bei Charakteristik 2", J. Reine Angew. Math. 307/308 (1979), 247-256. MR 80f:10020 Zbl 0396.12019

[Arason 2003] J. K. Arason, "Generators and Relations for $W_{q}(K((S)))$ ”, manuscript, 2003.

[Aravire and Jacob 1996] R. Aravire and B. Jacob, "Versions of Springer's theorem for quadratic forms in characteristic 2", Amer. J. Math. 118:2 (1996), 235-261. MR 97d:11064 Zbl 0853.11028

[Aravire and Jacob 2004] R. Aravire and B. Jacob, "The Milnor sequence for $W_{q} \mathscr{F}(x)$ in characteristic 2 when $\mathscr{F}$ is perfect.", pp. 1-17 in Algebraic and arithmetic theory of quadratic forms (Talca and Pucón, Chile), edited by R. Baeza et al., Contemporary Mathematics 344, American Math. Soc., Providence, RI, 2004. MR 2005f:11064 Zbl 1092.11022

[Lam 1973] T. Y. Lam, The algebraic theory of quadratic forms, Benjamin, Reading, MA, 1973. Revised reprint, 1980. MR 53 \#277 Zbl 0437.10006

[Milnor 1970] J. Milnor, "Algebraic K-theory and quadratic forms", Invent. Math. 9 (1970), 318344. MR 41 \#5465 Zbl 0199.55501

[Scharlau 1972] W. Scharlau, "Quadratic reciprocity laws", J. Number Theory 4 (1972), 78-97. MR 45 \#1835 Zbl 0241.12005

[Schilling 1950] O. F. G. Schilling, The theory of valuations, Mathematical Surveys 4, American Math. Soc., New York, 1950. MR 13,315b Zbl 0037.30702

Received February 25, 2005. Revised September 15, 2005.

\author{
ROBERTO ARAVIRE \\ DePartamento de Ciencias Físicas y Matemáticas \\ UNIVERSIDAD ARTURO PRAT \\ CASILLA 121 \\ IQUIQUE \\ CHILE \\ raravire@unap.cl \\ BILL JACOB \\ DEPARTMENT OF MATHEMATICS \\ UNIVERSITY OF CALIFORNIA SANTA BARBARA \\ SANTA BARBARA, CA 93106 \\ UNITED STATES \\ jacob@math.ucsb.edu
}

